\title{
TITLE:
}

\section{GLOBAL LOGIC RESULTING FROM DISEQUILIBRATION PROCESS}

$\operatorname{AUTHOR}(\mathrm{S})$ :

GUNJI, Yukio-Pegio

\section{CITATION:}

GUNJI, Yukio-Pegio. GLOBAL LOGIC RESULTING FROM DISEQUILIBRATION PROCESS. 数 理解析研究所講究録 1994, 885: 178-205

ISSUE DATE:

1994-09

URL:

http://hdl.handle.net/2433/84288

RIGHT: 


\section{GLOBAL LOGIC RESULTING FROM DISEQUILIBRATION PROCESS}

\section{Yukio-Pegio GUNJI}

Depatrment of Earth \& Planetary Sciences, Faculty of Science, and Graduate School of Sicence \& Technology, Kobe University Nada, Kobe 657 JAPAN

Describing a system in which internal detection or observation proceeds at a finite velocity is always destined to end up with a form of self-contradiction. For any formal language for such a description must assume that the velocity of observation propagation or VOP be infinity. In the present paper, we propose a self-referential scheme intended for formally describing a system exhibiting the process of disequilibration propagating at a finite VOP, and find that a global logic can emerge from local disequilibration. Conservative cellular automata of Margolus type, for instance, enable disequilibration to be replaced by such a process that the number of particles is not conserved globally while appearing to be conserved by local observers. One cannot determine local rules universally. Nevertheless, global logic emerges as a result of the dynamics of a one-to-many type mapping. This is a fundamental aspect of natural languages or communication relevant to natural life and intelligence.

\section{Introduction}

Describing the time evolution of an interacting many-element system usually forces us to employ a set of local rules claimed to be valid over the entire space. Such a globality of local rules suggests the existence of a kind of field, rendering both local and global inrteractions feasible in a mutually consistent manner. However, it is also suggested that local description often conflicts with its global counterpart in the realm of biology. One attempt for escaping the conflict is appraisal of hierarchical structure. This perspective naturally raises a question on the origin of hierarchy in life, evolution and intelligence, a lasting battle field between holism and reductionism.

Antagonism between local and global description originates in the aspect that one cannot have local rule that are claimed to be universal at the same time. This universality involves the assumption that the velocity of observation propagation (VOP) be set to be infinite. Once the finiteness of VOP is legitimately noted, one comes to face the disparity between the global feature generated by those local rules and those global features conflicting with the rules. Langevin equations, for instance, are an example exhibiting such a conflict, in which local rules embodied in the deterministic part show marked and conflicting contrast to the stochastic part. The larger the velocity of observation propagation is, the more effective the stochastic term would become in the time evolution of the system called dissipative structures. If one replaces the deterministic term by a chaotic map, a coupled map lattice can well approximate a system with a finite VOP (Crutchfield \& Kaneko, 1987; Kaneko, 1989). An artificial enzymatic neuron (Conrad, 1985; Kirby \& Conrad, 1986) would also do the similar approximation. For the relationship between chaotic map and diffusion emulates the relationship between intra- and inter-cellular computation. If 
one understands that the assumption of an infinite vop arises from discarding intra-cellular computation, a coupled map lattice may be an approximation to a system with a fininte VOP.

However, describing a system with a finite VOP or not forgetting intra-cellular computation leads to a paradox or unprogrammability. This differs from dissipative structures that could be defined definitely. A Resolution out of such a paradox might be either an approximate description or a description by the logic embedding paradoxes in an emergent state. Varela tried the latter type of formulation for life (Varela, 1979), and came up with autopoiesis (Maturana \& Varela, 1980). A contradictory state such as $\alpha=7 \alpha$ in the two-valued logic (the primary algebra) is embedded in Varela's extended algebra, while the extended algebra no longer admits the principles of the excluded middle. Although paradoxes in the primary algebra have been removed in the extended algebra, actual identification of the process of removing paradoxes is missing there. The present incapability of identification is similar to the lack of intra-cellular computation in the coupled map lattice.

Chaotic dynamics involves indeterminacy with respect to the halting problem (Nicolis, 1991). It implies that whether or not the time evolution through iterating a chaotic map be attracted into a stable fixed point for any set of initial and boundary conditions is not determinate due to the presence of Lyapunov exponent greater than zero. In other words, if the history or the actual trajectory is assumed to carry semantic values, chaotic attractors entail indeterminacy. Indeed, some chaotic maps like a map expressing Belousov Zapotinsky reaction induce an order (Matsumoto \& Tsuda, 1983; Matsumoto, 1984), but some do not. Behavior of the evolution through chaotic maps is completely determinate in a state space. This implies that any changes in state space needs to be controlled by an order parameter. Even if one may find a strange chaotic attractor that may well emulate an organism, it has to be examined how the state space evolved in the first place, from simple to complex. Therefore, one is destined to examine the origin of life again.

While describing a system with a finite VOP leads to a paradox, it is a necessary condition for life and complexity but not sufficient. This is the reason why some chaotic dynamics induces an order even under the presence of perturbations but some do not. Moreover, self-repairing or self-organizing stability and evolvability, in addition to indeterminacy, are regarded as essential features of biological systems (Fleishaker, 1989). We note that if one defines a paradox as a form of dynamics by employing a one-to-many type mapping under a given logical constraint, one can formalize a system with a fininte VOP and accordingly to comprehend self-organization processes and evolvability (Gunji \& Nakamura, 1991; Gunji \& Konno, 1991; Gunji, 1992, 1993a,b). A system with a finite vop exhibits its own dynamics as much as a programmbale machine does, in the latter of which however VOP is infininte. Hence, an external observer toward a system with a finite VOP finds that removal of preceding paradox causes subsequent another paradox in the manner that removal of paradox is destined to fail every time(Matsuno, 1989). Such a system looks as if it increases the diversity of its own rules and enhances the specificity of actual rules with time through repetition of limited rules (Gunji, 1993b). This is an aspect of self-organizing process featuring evolvability.

In this paper, a relationship between a self-referential system (Gunji, 1993b) and a system with a finite VOP is formally 
examined. In the end, we will emphasize the role of a one-tomany type mapping embodying a paradox in general state space. The one-to-many type mapping differs from a chaotic attractor in that such features of self-organizing processes as tending toward a stable state in some time and moving away. from it in other times are common to any system with a finite VOP. Since the finiteness of VOP is found in any material system, selforganizing capability would also be found in more or less all material systems. In this context, evolvability is intrinsic to any material system in its own right. We will demonstrate an operation of disequilibration process with the use of selfreferenital systems comprising Margolus cellular automata (Margolus, 1984; Toffoli \& Margolus, 1987). When the system with a finite VOP below which self-consistency cannot be maintained any more is the case, a global logic that cannot be reduce to local ones will be found. Hierarchical structures will arise through interference between local and global description.

\section{Perspective of an Internal Observer}

It has been argued that the phenomenon of life is described as a self-referential form because no form in organisms can be separated from its function. The ambiguity in the separation of an operator and an operand is originated from the aspect of a finite VOP. (Matsuno, 1989; Gunji \& Nakamura, 1991; Gunji \& Konno, 1991). The finite VOP is compared to the velocity of reading sentences by a reading subject, in which each page of a book is turned by the reader or the internal observerbefore he has finished reading the page. This indicates an instance of a finite VOP (Gunji, 1993). While formal description of interactions should be expressed from the perspective of an internal observer, an external observer appears to be distinguished from the internal counterpart. This is because the resulting description involves the assumption of an infinite VOP on the part of external observer on the one hand and at the same time the internal observer reads pages (interacts with them) at a finite VOP (Gunji \& Nakamura, 1991). As a result, the external observer cannot identify the correspondence table between words and their meanings that theinternal observer has even if the latter has its own correspondence table.

When one describes a form of interaction among components in a system, it is always assumed that interactions are communicated at an infinite VOP. In terms of the category theory, the assumption of an infinite VOP can be compared to that of the limit of a category $c$, because the limit represents the presence of a universal observer. That limit introduces the products. Also, extending the idea of the limit between categories, one can obtain the definition of the exponential and the isomorphism $\mathrm{C}(A \times B, C) \simeq C\left(B, C^{A}\right)$. It implies that any interaction $\phi: A \times B \rightarrow C$ is expressed by the form of $\phi=e v \circ\left(i_{A} \times \hat{\phi}\right)$ where ev: $A \times C^{A} \rightarrow C, \quad \hat{\phi}: B \rightarrow C^{A}$, and $i_{A}: A \rightarrow A$ representing an identity. It also implies that any interaction is articulated into the form of identifying an input $(\hat{\phi})$ and processing it $(\mathrm{ev})$. Insofar as one accepts an identification process proceeding at a finite VOP, one obtains a paradox or a fixed point (Gunji \& Nakamura, 1993).

Let $\hat{\phi}$ be a tactile process of an enzyme and ev be a transformation applied to an active pattern in an enzymatic neuron (Conrad, 1985). If a tactile process proceeds at an infinite VOP, one can express a series of reactions in an enzymatic neuron in the language of a sequential machine. That 
is due to the commutability of $\phi=\mathrm{ev}^{\circ}\left(\mathrm{id}_{A} \times \hat{\phi}\right)$ for any pair of an input $\left(a^{t}\right)$ and an internal state $\left(b^{t}\right)$ in $A \times B$. The resulting active pattern at $t+\Delta t$ in $C$ is expressed as $c^{t+\Delta t}=\phi\left(a^{t}, b^{t}\right)$. Once we examine the tactile process proceeding over a finite time $\Delta t^{\dot{ }}\left(\Delta t>\Delta t^{\prime}\right)$, the interaction will turn out to have the form $c^{t+\Delta t}=\hat{\phi}\left(b^{t}\right)\left(a^{t}\right)=f^{t+\Delta t}\left(a^{t}\right)$, where $f^{t+\Delta t} \in C^{A}$. Expressing the tactile process as

$$
f^{t+\Delta t}=\hat{\phi}\left(b^{t}\right)
$$

however, is not sufficient enough for the process proceeding at a finite VOP. An internal observer sitting on an enzyme cannot control the timing of receiving an input. This can lead to receiving another input in the midst of the preceding tactile process proceeding at a finite VOP. Therefore, the internal observer has to compute the tactile process in the presence of a small deviation of the timing $\left(\Delta t_{1}<\Delta t^{\prime}\right)$ for receiving an input. This requires

$$
f_{1} t+\Delta t^{\prime}=\hat{\phi}_{1}\left(b^{t+\Delta t}\right)
$$

Eq-(2-2) also implies that a small perturbation to an internal state (operand) comes to affect or change the operator, $\hat{\phi}$. Similarly, controlling the tactile process requires the expression like (2-2) for any small time duration $\Delta t_{i}$, and finally we obtain

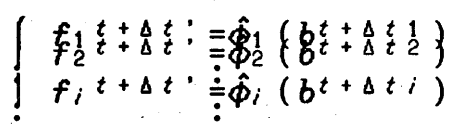

to define the timing of receiving for any ascending accuracy, $\Delta t_{1}>\Delta t_{2}>>\cdots>\Delta t_{i}>>\cdots$. That is an infinite regression. In short, a system proceeding at a finite VOP entails the observation that one has to regard a bundle of trajectories (Eq-(2-3)) as a trajectory in the state space of $\phi: A \times B \rightarrow C$. In other words, programming of a system proceeding at a finite VOP leads to the measurement problem in which one has to identify the neighborhood of a single state as a specific single state. However the neighborhood is ill-suited in the sense of structural stability. The measurement problem of a system proceeding at a finite VOP is expressed as follows. If an internal observer uses a logic of which any statement is programmable (i.e., any propostion can be proved either $A$ or not $A$, where $A$ is any semantical value), a logic itself involves the assumption of an infinite VOP. The reason why it leads to an infinite regression, is that the aspect in which an internal observer measures at a finite VOP is describable under the assumption that he measures at an infinite VOP. In order to cope with the process posessing such an infinite regression, the internal observer has to choose a specific infinite sequence $\quad \beta=\left(b^{t+\Delta t 1}, b^{t+\Delta t 2}, \ldots, b^{t+\Delta t i}, \ldots\right) \in \mathrm{B}=\mathrm{B} \times \mathrm{B} \times \cdots$ (an infinite product) and identify its meaning. Define an infinite set $B=\left\{\beta_{0}, \beta_{1}, \ldots, \beta_{k}, \ldots\right\}$ and the set of its meanings as $B$. Also, define $\hat{\psi}_{1}: B \rightarrow B^{B}$ such that

$$
\mathrm{B} \stackrel{\Delta \longrightarrow}{\longrightarrow} \mathrm{B} \times \mathbf{B} \stackrel{i \mathrm{~d}_{\mathrm{B}} \times \hat{\psi}_{1}}{\longrightarrow} \mathrm{B} \times B^{\mathrm{B}} \stackrel{\mathrm{eV}}{\longrightarrow} B
$$

where $\Delta$ represents a diagonal map. One can choose a specific infinite sequence and identify its meaning if one knows the 
correspondence table between $B$ and $B^{B}$. It implies that $B^{B}$ involves no extraneous element with respect to $\hat{\psi}_{1}(\mathrm{Gunji} \&$ Nakamura, 1993). Finally, for any $\beta$ there exists $\beta^{\prime}$ such that $g(\beta)=\hat{\psi}_{1}\left(\beta^{\prime}\right)(\beta)$ in terms of any $g: B \rightarrow B$, and we obtain

$$
b=\hat{\psi}_{1}\left(\beta^{\prime}\right)(\beta)
$$

for any $b$ in $B$ (Lawvere, 1969). Note that the measurement in choosing $\beta$ leads to a fixed point because $\hat{\psi}_{1}$ has a selfreferential property. If one assumes that one can identify a specific infinite sequence of $\hat{\phi}_{1}, \hat{\phi}_{2}, \ldots, \hat{\phi}_{i}, \ldots$ and define an infinite product map $\hat{\phi}=\left(\hat{\phi}_{1}, \hat{\phi}_{2}, \ldots, \hat{\phi}_{i}, \ldots\right): \mathbf{B} \rightarrow \mathbf{R}$, where $\mathbf{R}=C^{A} \times C^{A} \times \cdots$ (an infinite product), the measurement problem appears as that of in choosing an infinite sequence of $\gamma=\left(f_{1} t^{t} \Delta t^{\prime}, f_{2} t^{+} \Delta^{\prime}, \ldots\right.$, $\left.f_{i}{ }^{t+\Delta t^{\prime}}, \ldots\right)$ in a set $R$. Therefore, like the measurement problem of choosing $\beta$, we obtain the correspondence table between $R$ and $\left(C^{A}\right)^{R}$ as

$$
\mathbf{R} \stackrel{\Delta}{\longrightarrow} \mathbf{R} \times \mathbf{R} \stackrel{i d_{R} \times \hat{\psi} 2}{\longrightarrow} \mathbf{R} \times\left(C^{A}\right)^{R} \stackrel{e v}{\longrightarrow} C^{A},
$$

and for any $\gamma$ there exits $\gamma^{\prime}$ such that $h(\gamma)=\hat{\psi}_{2}\left(\gamma^{\prime}\right)(\gamma)$ in terms of any $h: R \rightarrow C^{A}$. Then for any $f$ in $C^{A}$, we obtain

$$
f=\hat{\psi}_{2}\left(\gamma^{\prime}\right)(\gamma) \text {. }
$$

In this framework, we have assumed the commutable diagram,

$$
\left.\underset{\Phi}{\mathbf{P}} \stackrel{\hat{\psi}_{2}\left(\gamma^{\prime}\right)(\gamma)}{\mathbf{B} \hat{\psi}_{1}\left(\beta^{\prime}\right)(\beta)}\right|_{B} ^{A} .
$$

The morphisms $R \rightarrow C^{A}$ and $B \rightarrow B$ involves the measurement problem owingto a finite VOP. Also, $\Phi: B \rightarrow R$ is surjective because now we assume that $\phi$ results from coding a natural system and then it involves the measurement problem. Therefore, in choosing any $f$ in $C^{A}$, for any a in $A$,

$$
\begin{aligned}
f(\alpha) & =\hat{\psi}_{2}\left(\gamma^{\prime}\right)(\gamma)(\alpha) \\
& =\hat{\psi}_{2}\left(\gamma^{\prime}\right) \circ \dot{\phi}(\beta)(\alpha) \\
& =\hat{\phi}^{\circ} \hat{\psi}_{1}\left(\beta^{\prime}\right)(\beta)(\alpha) .
\end{aligned}
$$

It implies that there exists $b$ in $B$ such that $b=\hat{\psi}_{1}\left(\beta^{\prime}\right)(\beta)$. Finally, for any $a$ in $A$ there exists $b$ in $B$ such that

$$
f(a)=\hat{\phi}(b)(a)
$$

We find that the measurement problem arising from a finite VOP leads to that $\hat{\phi}$ must be an onto mapping whether $A, B$ and $C$ are finite or infinite sets. It is clear that Eq-(2-10). leads to a paradox or a fixed point $\hat{\phi}(b)\left(a^{*}\right)$ such that

$$
\hat{\phi}(b)\left(a^{*}\right)=h \hat{\phi}(b)\left(a^{*}\right) \text {, }
$$

because for any $g: A \rightarrow C$ one can find $a^{*} \in A$ such that $g\left(a^{*}\right)=h \hat{\phi}(b)\left(a^{*}\right)$ with the use of any function $h: C \rightarrow C$ (Lawvere, 1969). It implies that one cannot determine a unique rule $\hat{\phi}$ in describing a system proceeding at a finite VOP under the assumption of an infinite VOP.

While $\hat{\phi}: B \rightarrow C^{A}$ can be identified whether or not it is an onto 
mapping, it must always be an onto mapping in sofar as one takes the measurement or a finite VOP into consideration. Hence the ambuigity in $\hat{\phi}$, which may be both an onto mappig and not at the same time can be no longer described in terms of a map. Then, we need the notation of a one-to-many type mapping $\langle\phi\rangle$, a symbol surrounded by a bracket. If we write the mapping as

$$
c^{t+\Delta t}=\langle\phi\rangle\left(a^{t}, b^{t}\right),
$$

it implies that there exists $b^{*}$ for $b^{t}$ such that $c^{t+\Delta t}$ is proved to be both $\phi\left(a^{t}, b^{t}\right)=\hat{\phi}\left(b^{*}\right)\left(a^{t}\right)$ and $h \hat{\phi}\left(b^{*}\right)\left(a^{t}\right)$ for any $h: C \rightarrow C$.

It has been argued that a system proceeding at a finite V.OP is destined to be described as a one-to-many type mapping (Matsuno, 1989; Gunj1 \& Nakanura, 1991; Gunji \& Konno, 1991; Gunji 1992), because it leads to a paradox (Gunji, 1992) or a fixed point (Gunji, 1993a,b; Gunji \& Nakamura, 1993). On the other hand, chaotic dynamics involves an indeterminacy in terms of Lyapunov exponent (Nicolis, 1991). Achaotic attractor is in fact one expression of a one-to-many type mapping. This observation involves the assumption that one can regard a bundle of trajectories as a trajectory in the sense of coarse-graning. Though it sounds trivially all right, our formalization of a system with a finite VOP suggests that it cannot be. While the commutable diagram (2-8) represents that the coarse-graining makes us choose a specific state space, the commutability leads to a paradox $(2-11)$ in its own right. A one-to-many type mapping results in a fixed point. Hence, we emphasize that a one-to-many type mapping in a system proceeding at a finite VOP is not described as a specific interpretation; the coarse-graining (Fig. 1).

However, the evolution of a system proceeding at a finite VoP does not actualize under no constraint. An external observer finds not only a paradox in this aspect but finds that the subsequent resulting removal of the resulting paradox is always destined to fail (Matsuno, 1989; Gunji, 1993b), because the internal observer can comprehend even an indefinite input or information in spite of non-existence of his own correspondence table. Note that a paradox appears when one describes an interaction while assuming that the internal observer has his own corresponding table between an input information and its meaning (1.e. enzyme switch) in spite of no objective corresponding table available to the external observer. This assumption implies that a system with a finite VOP is described in terms of a formal system featuring an infinite VOP. The idea of a system proceeding at a finite VOP lets us acknowledge that the system generates its own paradox and then removes it. That is the process of disequilibration.

Gunji (1993a, b) proposed a self-referential system which exhibits disequilibration with a finite VOP and demonstrated the breaking of a one-to-many type mapping $\langle\phi\rangle$ into a forward $(\phi)$, a backward $\left(\phi^{-}\right)$and a contradictory $\left(\phi_{c}\right)$ map. This formulation can be used even in the above context. Now $\hat{\phi}$, the transpose of $\phi$, is an onto mapping under a finite VOP. We can make this constraint weaker. Define that for any $(a, f) \in A \times C^{A}$ there exists $b \in B$ such that $f(a)=\hat{\phi}(b)(a)$. It does not imply $f=\hat{\phi}(b)$. We call this a weak self-referential property of $\hat{\phi}$. Therefore, there exists an onto map $\Gamma: A \times B \rightarrow A \times C^{A}$ such that $\operatorname{ev}^{\circ} \Gamma=\operatorname{ev}^{\circ}\left(i_{A} \times \hat{\phi}\right)=\phi$. Hence; if for any $k$, $k^{\prime}: C \rightarrow X$ with any $X, k^{\circ} \phi=k^{\prime} \circ \phi$, then $k=k^{\prime}$, because $k^{\circ} \circ \mathrm{ev}^{\circ} \Gamma=k^{\prime} \circ \mathrm{ev} \circ \Gamma$, $k \circ \mathrm{ev}=k^{\prime} \circ \mathrm{ev}$ ( $\Gamma$ is an onto-map) and then $k=k^{\prime}(\mathrm{ev}$ is also an ontomap). It implies that there exists an onto map $A \times B \rightarrow C$. Let $\phi_{c}$ be 
an onto map $\phi_{C}: A \times B \rightarrow C$. The aspect defined here implies that one cannot distinguish $\phi$ from $\phi_{c}$ and it entails a one-to-many type mapping $\langle\phi\rangle$. In order to describe such a paradoxical condition, we introduce the symbol $\sim$ instead of the symbol $=$, and express the condition as

$$
\phi\left(a^{t}, b^{t}\right) \sim \phi_{c}\left(a^{t}, b^{t}\right) .
$$

An idea of disequilibration leads to that one cannot determine either $\phi$ or $\phi_{c}$ a priori but can only a posteriori. This process means that if the state at instant $t$ is definite as $b^{t}$, the state at instant $t+\Delta t$ can be proved to be both $\phi\left(a^{t}, b^{t}\right)$ and $\phi_{c}\left(a^{t}, b^{t}\right)$, then

$$
b^{t} \rightarrow\left[\phi\left(a^{t}, b^{t}\right) \wedge \phi_{c}\left(a^{t}, b^{t}\right)\right]
$$

in a Booelan logic form for the sake of convenience. Hence it also implies that

$$
\left[>\phi\left(a^{t}, b^{t}\right) \vee>\phi_{c}\left(a^{t}, b^{t}\right)\right] \rightarrow>b^{t} .
$$

We regard $7 \phi\left(a^{t}, b^{t}\right)$ as $\phi_{c}\left(a^{t}, b^{t}\right)$, and $7 \phi_{c}\left(a^{t}, b^{t}\right)$ as $\phi\left(a^{t}, b^{t}\right)$, then the form $(2-15)$ can be replaced by $\left[\phi\left(a^{t}, b^{t}\right) \vee \phi_{c}\left(a^{t}\right.\right.$, $\left.\left.b^{t}\right)\right] \rightarrow>b^{t}$. We distinguish the statement a priori from that a posteriori, by way of the scheme that $\phi\left(a^{t}, b^{t}\right) \wedge>\phi\left(a^{t}, b^{t}\right)=\phi\left(a^{t}\right.$, $\left.b^{t}\right) \wedge \phi_{c}\left(a^{t}, b^{t}\right)$ a priori but $\phi\left(a^{t}, b^{t}\right) \vee>\phi\left(a^{t}, b^{t}\right) \neq \phi\left(a^{t}, b^{t}\right) \vee \phi_{c}\left(a^{t}\right.$, $\left.b^{t}\right)$ a posteriori. Then, while the statement (2-15) is ill-suited, this is not the statement that is always true. We here find that the statement (2-15) involves an act of choice a posteriori. It implies that if one determines the state at instant $t+\Delta t$ as $\phi_{c}\left(a^{t}, b^{t}\right)\left(\right.$ resp. $\left.\phi\left(a^{t}, b^{t}\right)\right)$ there exists $b^{\prime}{ }^{t}$ such that

$$
\phi_{c}\left(a^{t}, b^{t}\right)=\phi\left(a^{t}, b^{t} t\right)
$$

(resp. $\left.\phi\left(a^{t}, b^{t}\right)=\phi_{c}\left(a^{t}, b^{t} t\right)\right)$. Note that we distinguish the state a priori from that a posteriori. Since the form (2-13) implies both $\phi_{c}\left(a^{t}, b^{t}\right)=\phi\left(a^{t}, b^{t}\right)$ and $\phi_{c}\left(a^{t}, b^{t}\right) \neq \phi\left(a^{t}, b^{t}\right)$, the statement (2-14) means that the principles of the excluded middle does not hold a priori. The statement (2-15), however, implies that one can choose $7 \phi\left(a^{t}, b^{t}\right)$ or $7 \phi_{c}\left(a^{t}, b^{t}\right)$ a posteriori. We can distinguish the state a priori from that a posterioi by starting from the ill-suited statement (2-14).

Eq-(2-16) implies that for any $(\alpha, c)$ such that $c=\Phi_{c}(\alpha$, $b) \neq \phi(a, b)$, there exists $b^{\prime}$ such that $c=\phi\left(a, b^{\prime}\right), b \neq b^{\prime}$. Therefore, we can define $\phi^{-}: A \times C \rightarrow B$ such that for any $\left(a^{t}, b^{t}\right) \in A \times B$

$$
7 \phi\left(a^{t}, b^{t}\right)=\phi\left(a^{t}, \phi^{-}\left(a^{t}, \phi_{c}\left(a^{t}, b^{t}\right)\right)\right) .
$$

The articulation of distinction between a priori and a posteriori is embodied through the introduction of both a forward ( $\phi$ and $\phi_{c}$ ) and a backward map $\left(\phi^{-}\right)$due to $b^{\prime t}=\phi^{-}\left(a^{t}, c^{t+\Delta t}\right)$. Finally, because of the condition $(2-13), \phi\left(a^{t}, b^{t}\right) \sim \phi_{c}\left(a^{t}, b^{t}\right)=7 \phi\left(a^{t}, b^{t}\right)$ and Eq-(2-17), we obtain the form of a one-to-many type mapping as

$$
<\phi>\left(a^{t}, b^{t}\right)=<\phi>\left(a^{t}, \phi^{-}\left(a^{t}, \phi_{c}\left(a^{t}, b^{t}\right)\right)\right) .
$$

It cannot be programmable. However, if when we rewrite Eq-(2-18) to 


$$
\begin{array}{ll}
c_{x} t+\Delta t=\phi\left(a^{t}, b^{t}\right), & (2-19 a) \\
b_{x}{ }^{t}=\phi^{-}\left(a^{t}, \phi_{c}\left(a^{t}, b^{t}\right)\right), & (2-19 b)
\end{array}
$$

the-one-to-many type mapping can be made programmable with the use of the transition from $b_{x}{ }^{t}$ to $c_{x}{ }^{t+\Delta t}$. It is clear that a paradox follows if one removes all brackets from the form (2-18). In short, we can break the form of a fixed point down into Eq$(2-19 a)$ and $(2-19 b)$.

In the previous paper, we poposed a system of the form similar to Eq-(2-19), called the self-referetial system. We shall call the system (2-19) also the self-referential system. In the self-referential system, the time transition expressed in (2-19) does by no means follow the transition defined by the map $\phi$. Therefore, it looks as if transition rules are perpetually changed as time progresses. Also, if one defines a local rule such as $\phi: A^{3} \rightarrow A$, one finds either that $\phi$ no longer asserts for universality or that the local rule is of a one-to-many type mapping. At this point, it is reasonable to suppose that if $\phi=\phi_{c}$, the system, has no paradox conditioned by an infinite VOP. The smaller the VOP, the larger the metric distance between $\phi$ and $\phi_{c}$. Hence we can estimate the behavior of a system in terms of VOP. In the next chapter we shall estimate it for the case of the Margolus cellular automata (Margolus, 1982).

\section{Self-reference in conservative system}

\section{3-1. local universal rule in Margolus automata}

In this section, we replace the consistency of a local rule by the conservation of the number of particles in the scheme of cellular automata. If a local rule has the universality in the space, the number of particles counted at each a unit time duration is conserved. It means that the local logic of the conservation law is consistent with the global logic. As far as an infinite VOP were taken for granted, the global and local logic would be tautological. A system proceeding at a finite VOP is destined to lose the universality of a local rule, and then it is expected to exhibit disequilibrations.

To express a conservative system in the cellular automata, Margolus (1982) defined a local universal rule $f: \Omega^{2} \rightarrow \Omega^{2}, \Omega=\{0,1\}$ which has the invertibility, over a whole lattice space. If $f$ is defined in such a manner that $f(0,0)=(0,0), f(0,1)=(1,0)$, $f(1,0)=(0,1)$, and $f(1,1)=(1,1)$ [we call the rule the original Margolus CA], the number of particles denoted as 1 is conserved both locally and globally. A step-wise grid conversion (Fig. 2a) is also introduced to make a pair of lattice interact with the nearest pair. As a result, a local rule $f$ loses the universality in the strict sense because the time evolution of the state of a lattice requires the determinant of either grid 1 or 2 by which the position of operation $f$ is determined, and it requires both the position and the time step of the lattice.

A fixed point owing to the finite VOP is excressed in the form that a local universal rule cannot uniquely be determined. It, thus, requires the formal relation between the decidability of a local universal rule and the conservation law. We have to formulate a local universal rule corresponding to the Margolus CA without introducing the setp-wise grid conversion. Taking Margolus CA as the rule space,

$$
R_{M}=\left\{f \mid \Omega^{2} \rightarrow \Omega^{2}, f \text { is bijective }\right\},
$$


denote a rule $f \in R_{M}$ by the rule number \#(f) defined by

$$
\#(f)=<d_{\theta}, d_{1}, d_{2}, d_{3}>,
$$

such that, for $\forall(a, b) \in \Omega^{2}, d_{k}=f(a, b)$ and $k=2 a+b$. The rule $<0$, $2,1,3>$ denotes the rule exhibiting the time evolution as shown in Fig. 2A. The number of elements of $\mathrm{RM}_{\mathrm{M}}$ is $4 !=24$, and we identify a rule $f$ with $\bar{f}$ if $\#(f)=<d_{0}, d_{1}, d_{2}, d_{3}>$ and

$$
\#(\bar{f})=\left\langle\overline{d_{3}}, \overline{d_{1}}, \overline{d_{2}}, \overline{d_{\theta}}>\right.\text {, }
$$

where $\overline{d_{k}}=\zeta_{1}\left(d_{k}\right)$ such that $3=\zeta_{1}(0), 2=\zeta_{1}(1), \quad 1=\zeta_{1}(2), \quad 0=\zeta_{1}(3)$. Also identify a rule $f$ with $f$ if $\#(f)=\left\langle d_{0}, d_{1}, d_{2}, d_{3}\right\rangle$ and

$$
\#(f)=<a_{0}, a_{2}, a_{1}, a_{3}>,
$$

where $a_{k}=\zeta_{2}\left(d_{k}\right)$ such that $0=\zeta_{2}(0), 2=\zeta_{2}(1), 1=\zeta_{2}(2), 3=\zeta_{2}(3)$. As a result of this identification, the number of rules in Margolus $\mathrm{CA}$ is degenerated into ten types as shown in Fig. 3.

Now we define a local universal rule $\phi$ simulating a rule $f$ in Margolus CA if there exists a map $\phi: \Omega^{5} \rightarrow \Omega$ such that for any $\left(a_{i-1}{ }^{t-1}, \alpha_{i}{ }^{t-1}, a_{i-2}{ }^{t}, a_{i-1}{ }^{t}, \alpha_{i}{ }^{t}\right)=(\alpha, b, e, c, d) \in \Omega^{5}$ there exists $a_{i}{ }^{t+1}=\alpha \in \Omega$ such that

$$
\alpha=\phi(\alpha, b, e, c, d) .
$$

The number of arguments in $\phi$ can be decreased and the number 5 is the maximal number. It implies that there are at most two cases in determining the value of $\alpha=\alpha_{i}-1^{t+1}$ and $\beta=\alpha_{i} t+1$ : (i) one can determine whether $\left(\alpha_{i-1} t, \alpha_{i}{ }^{t}\right)=(c, d)$ results from the operation adopting the grid 1 or 2 (Fig. 2b). It leads that one can determine whether $\alpha=\pi_{2}$ of $(e, c)$ or $\alpha=\pi_{1}$ of $(c, d)$, where $\pi_{1}: \Omega^{2} \rightarrow \Omega$ (resp. $\pi_{2}$ ) is a projection map taking the first (resp. the second) component. Hence, if for given $\left(a_{i-1}{ }^{t-1}, a_{i}{ }^{t-1}\right)=(a, b)$ and $\left(a_{i-1}{ }^{t}, a_{i}{ }^{t}\right)=(c, d)$,

$$
(c, d)=f(a, b)
$$

and there does not exist $x, y \in \Omega$ such that

$$
c=\pi_{2} \circ f(x, a), d=\pi_{1} \circ f(b, y),
$$

one can see that $(c, d)=f(a, b)$ results from the operation along the grid 1. Otherwise, it results from the grid 2. (ii) If one cannot determine either grid 1 or 2 , one can obtain

$$
\pi_{1} \circ f(c, d)=\pi_{2} \circ f(e, c) .
$$

Note that the equation $\left(a_{i-1}{ }^{t}, a_{i}^{t}\right)=f\left(a_{i}-1^{t-1}, a_{i}{ }^{t-1}\right)$ can be rewritten to $\alpha_{i-1}{ }^{t}=f_{1}\left(\alpha_{i-1}{ }^{t-1}, \alpha_{i}{ }^{t-1}\right), \alpha_{i}{ }^{t}=f_{2}\left(\alpha_{i-1}{ }^{t-1}, a_{i}{ }^{t-1}\right)$ by using $f_{1}, f_{2}: \Omega^{2} \rightarrow \Omega$ defined by $f_{1}=\pi_{1}$ of and $f_{2}=\pi_{2} \circ f$. Finally, as far as one distinguish the condition of grid 1 [denoted by $\psi\left(a_{i}-1^{t-1}\right.$, $\left.\left.a_{i}{ }^{t-1}, \alpha_{i-1}{ }^{t}, \alpha_{i}{ }^{t}\right)\right]$ from that of grid 2, one can generally express a universal local rule as

$$
\begin{aligned}
& a_{i}{ }^{t+1}=\Phi\left(a_{i-1}{ }^{t-1}, a_{i}{ }^{t-1}, a_{i-2}{ }^{t}, a_{i-1}{ }^{t}, a_{i}{ }^{t}\right) \\
& =\left[\psi\left(\alpha_{i-1}{ }^{t-1}, \alpha_{i}{ }^{t-1}, \alpha_{i-1} t, \alpha_{i}^{t}\right) \rightarrow f_{2}\left(\alpha_{i-1}{ }^{t-1}, \alpha_{i}{ }^{t-1}\right)\right] \\
& \wedge\left[7 \psi\left(a_{i}-1^{t-1}, a_{i}{ }^{t-1}, a_{i-1} t, a_{i}{ }^{t}\right) \rightarrow f_{1}\left(a_{i}-1^{t-1}, a_{i}{ }^{t-1}\right)\right],
\end{aligned}
$$


where the symbol $\rightarrow, \wedge$, and $>$ represents Boolean operators, IMPLICATION, OR and NOT.

For exmaple, we can estimate a local universal rule corresponding to the original Margolus one, $\#(f)=<0,2,1,3>$, type 2. In this rule $(c, d)=f(a, b)$, iff $b=c$ and $a=d$. For given $\left(a_{i-1}{ }^{t-1}, a_{i}{ }^{t-1}\right)=(a, b)$ and $\left(a_{i-2} t, a_{i-1} t, a_{i}{ }^{t}\right)=(e, c, d)$, one can determine either $c=\pi 1 \circ f(a, b)$ or not if $d \neq e$. Because if $a=d$, one can find $(c, d)=f(a, b)$ and find that there is no $x \in \Omega$ such that $(e, c)=f(x, a)$ due to $e \neq a$ (i.e. grid 1). Likewise, if $a \neq d$, one can find $x \in \Omega$ such that $(e, c)=f(x, \alpha)$ due to $e=\alpha$ and (c, $d) \neq f(a, b)$ (i.e. grid 2). Therefore, if $a=d$ (resp. $a \neq d$ ), one can obtain $a_{i-1}{ }^{t+1}=\alpha$ such that

$$
\alpha=e(\operatorname{resp} . \alpha=d) \text {. }
$$

Also, one cannot determine either grid 1 or 2 (i.e. the grid can be proved to be both 1 and 2) if $d=e$. It implies, however, $d=\alpha=e$. Then one can obtain $\pi_{1} \circ f(c, d)=\pi 2 \circ f(e, c)=d=e$. Finally we obtain $\phi: \Omega^{3} \rightarrow \Omega$ such that

$$
\begin{aligned}
& \alpha_{i}{ }^{t+1}=\phi\left(\alpha_{i-1} t, \alpha_{i}{ }^{t-1}, \alpha_{i+1}{ }^{t}\right) \\
& =\left(\left(\alpha_{i}{ }^{t-1} \oplus \alpha_{i+1} t\right) \rightarrow \alpha_{i+1} t\right) \wedge\left(\left(>\left(\alpha_{i}{ }^{t-1} \oplus \alpha_{i+1} t\right)\right) \rightarrow \alpha_{i-1}{ }^{t}\right) \\
& =\left(\alpha_{i+1}{ }^{t} \wedge>a_{i}{ }^{t-1}\right) \vee\left(\left(\alpha_{i}{ }^{t-1 \rightarrow a_{i+1} t}\right) \wedge \alpha_{i}-1^{t}\right) \text {. }
\end{aligned}
$$

where the symbol $\oplus$ and $V$ represents Boolean operators, MODULO and OR. However, in this formulation there exists an ill-suited value of the triplet $\left(\alpha_{i-1}{ }^{t}, \alpha_{i}{ }^{t-1}, \alpha_{i+1}{ }^{t}\right)=(e, \alpha, d) \in \Omega^{3}$, because $f: \Omega^{2} \rightarrow \Omega^{2}$ on the one hand and $\phi: \Omega^{3} \rightarrow \Omega$ on the other. Altough it will be discussed in the later section, we note the simulatability of $\phi$ with respect to $f$. Now, for any configuration $\eta \in \Omega^{n}$ and the boundary condition $\xi \in \Omega^{2}, \overline{f_{k}}: \Omega^{n+2} \rightarrow \Omega^{n}, k=1,2$, and $\bar{\phi}: \Omega^{2}(n+2) \rightarrow \Omega^{n}$ are defined by the operation of $f$ and $\phi$ over the whole space $\Omega^{n+2}$ and $\Omega^{2}(n+2)$ respectively, where $\overline{f_{k}}$ represents the operation of $\bar{f}$ along the grid $k$. It is defined that the rule $\phi$ can simulate the behavior of the rule $f$ in the Margolus CA if for any $n \in \Omega^{n}$ and $\xi$, $\xi^{\prime} \in \Omega^{2}$,

$$
\bar{\phi}\left(\eta,\left(\overline{f_{k}}(\eta, \xi), \xi^{\prime}\right)\right)=\overline{f_{s}}\left(\overline{f_{k}}(\eta, \xi), \xi^{\prime}\right),
$$

where $s=2$ if $k=1$ (resp. $s=1$ if $k=2$ ).

For all ten types in the Margolus CA, one can determine a local universal rule for eight types as well as the original Margolus CA as shown in Table 1. Type 5 and 6 are the only rules such that $\mathrm{Eq}-(7)$ does not hold when the condition (6) is not satisfied. It can be easily proved if one classify the condition for a given $\left(\alpha_{i-1}{ }^{t-1}, \alpha_{i}{ }^{t-1}, \alpha_{i-2}{ }^{t}, \alpha_{i-1}{ }^{t}, \alpha_{i}{ }^{t}\right)=(\alpha, b, e, c$, d) $€ \Omega^{5}$. Finally one can find that for a given $\left(\alpha_{i}-1^{t-1}, \alpha_{i}{ }^{t-1}\right.$, $\left.a_{i-2}{ }^{t}, a_{i-1^{t}}, \alpha_{i}{ }^{t}\right)=(\alpha, a, 1-\alpha, 1-\alpha, 1-\alpha), \alpha \in \Omega, E q-(7)$ does not hold if $(\alpha, 1-\alpha)=f(1-\alpha, 1-\alpha)$ or $(1-\alpha, \alpha)=f(1-\alpha, 1-\alpha)$. From the assumption of a given configuration, $(1-\alpha, \quad 1-\alpha)=f(\alpha, \quad \alpha)$. Therefore, one cannot determine whether the time transition of $(1-\alpha, 1-\alpha)=f(\alpha, \alpha)$ follows grid 1 or grid 2 . As far as ( $\alpha, 1-$ $a)=f(1-a, 1-a) \quad(\operatorname{resp} . \quad(1-a, a)=f(1-a, 1-a)), \quad\left(a_{i}-1^{t+1}, a_{i}{ }^{t+1}\right)=$ $f(1-\alpha, 1-\alpha)=(\alpha, 1-\alpha)$ and $\alpha_{i}{ }^{t+1}=1-\alpha \quad\left(\right.$ resp. $\left.\alpha_{i}{ }^{t+1}=\alpha\right)$ if $(1-\alpha, 1-$ $\alpha)=f(\alpha, \alpha)$ follows the grid 1, and $\left(\alpha_{i}{ }^{t+1}, \alpha_{i+1}{ }^{t+1}\right)=f(1-\alpha, 1-$ $\alpha)=(\alpha, 1-\alpha)$ and $\alpha_{i}{ }^{t+1}=\alpha$ (resp. $\left.a_{i} t+1=1-\alpha\right)$ if $(1-\alpha, 1-\alpha)=f(\alpha, a)$ follows the grid 2 . It is easy to find that the condition ( $\alpha$, 1$\alpha)=f(1-\alpha, 1-\alpha)$ or $(1-\alpha, \alpha)=f(1-\alpha, 1-\alpha)$ is satisfied in the rule $\#(f)=\langle 3,0,2,1>$; $<3,2,0,1>;<3,0,1,2\rangle ;<3,1,0,2\rangle$ (if $\alpha=0)$; and $\langle 1,2,3,0\rangle ;\langle 1,3,2,0\rangle ;\langle 2,1,3,0\rangle ;\langle 2,3,1,0\rangle$ 
(if $\alpha=1$ ). They all belong to type 5 or 6 (Fig. 3 ).

For a rule of the Margolus CA of which there exists a local universal rule, one can define a rule space in which local universal rules generally have no weak self-referential property while there exists a local rule with weak self-referential property. In the rule space, we estimate the significance of the weak self-referential property and can demonstrate the disequilibration process such that the contradiction arising from the process as to remove the contradiction can be by no means removed and the process perpetually continues.

3-2. Self-contradiction and conservation of the number of particles

For a given local universal rule, $\phi: \Omega^{n} \rightarrow \Omega, n \leq 5$, which can simulate the rule $f$ in Margolus CA, we define the rule space $R_{\mathfrak{u}}(f)$ that involves a specific rule which can simulate a Margolus rule by

$$
R_{\mathfrak{n}}(\boldsymbol{\rho})=\left\{\phi: \Omega^{n} \rightarrow \Omega\right\} \text {. }
$$

It is defined that the rule $f$ constitutes the rule space. $R_{k}(f)$. In this section, we concentrate on the rule space $R\langle 0213\rangle$ which is constituted by the original Margolus rule of which the number of particles is locally coserved and then one can find a global conservation law with respect to the number of particles. In this rule space, the rule which has a weak self-referential property has a local conservation law. Therefore removing the contradiction arising from the pursuit of logical consistency in spite of the self-reference can be replaced by removing the contradiction arising from the pursuit of local conservation of the number of particles in spite of a finite VOP.

The rule of $\#(f)=<0,2,1,3>$ shows a symmetirc feature, and then we here define $R\langle 0213\rangle=\left\{\phi \mid \Omega^{3} \rightarrow \Omega, \phi(\alpha, b, c)=\phi(c, b, \alpha)\right\}$, where $a, b, c \Subset \Omega$. The $\phi: \Omega^{3} \rightarrow \Omega$ defined by $E q-(3-8)$ is expressed as

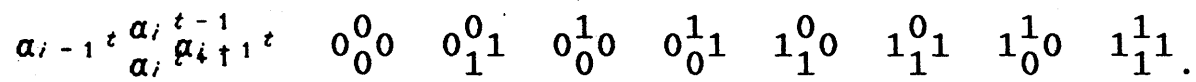

However, $\left(a_{i-1^{t}}, \alpha_{i}{ }^{-1}, \alpha_{i+1} t\right)=(0,1,0)$ and $(1,0,1)$ are illsuited values as arguments because such configurations cannot be found in the time evolution of the original Margolus rule. The simulatability defined by Eq-(3-11) admits that the rule $\phi$, defined as in the form (3-13) except for $\phi(0,1,0)$ and $\phi(1,0$, $1)$, can simulate the original Margolus rule irrespective whether $\phi(0,1,0)$ and $\phi(1,0,1)$ equals to 1 or 0 . Consequently, there exist four rules of $\phi$ that can simulate the original Margolus rule.

When the expression of $\phi$ can be rewritten to $a_{i}{ }^{t+1}=\phi(x$, $\left.a_{i}{ }^{t-1}, y\right), \quad x=a_{i}-1^{t} \oplus \alpha_{i}{ }^{t-1}$ and $y=\alpha_{i}+1^{t} \oplus \alpha_{i}{ }^{t-1}$, any rule which can simulate the original Margolus rule is expressed as

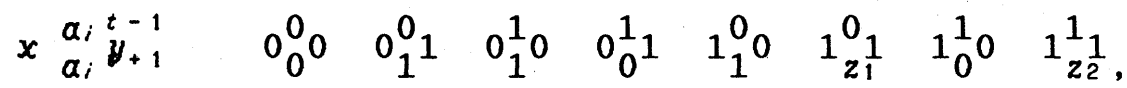

where $z_{1}$ and $z_{2}$ are either 0 or 1 , respectively. Finally we obtain the local universal rule which can simulate the original Margolus rule with a weak self-referential property in the form of $a_{i}{ }^{t+1}=\phi\left(x, a_{i}{ }^{t-1}, y\right)$. Because one need not have to pay attention to $\phi(1,0,1)$ and $\phi(1,1,1)$ which have nothing to do 
with the simulatability, one finds that for any triplet of $(a, b$, $c) \in \Omega^{3}$ except for $(1,0,1)$ and $(1,1,1), \phi(a, b, c) \neq \phi(a, 1-b$, $c)$. It is equivalent to the weak self-referential property(Gunji, 1993b).

When the rule number of $\phi \in R\langle 0213\rangle, \#(\phi)$, is defined by

$$
\begin{aligned}
& \#(\phi)={ }_{k} \underline{Z}_{0} d_{k} 2^{k}, \\
& d_{k}=\phi(\alpha, b, c), \\
& k=4(\alpha \oplus b)+2 b+(b \oplus c),
\end{aligned}
$$

with $a, b$ and $c \in \Omega$, the rule number of the rules with the weak self-referential property in the form of $\alpha_{i}{ }^{t+1}=\phi\left(x, \alpha_{i}{ }^{t-1}, y\right)$ are $22\left(z_{1}=z_{2}=0\right), 54 \quad\left(z_{1}=0, z_{2}=1\right), 150 \quad\left(z_{1}=1, z_{2}=0\right)$ and $182 \quad\left(z_{1}=z_{2}\right.$ $=1)$. They ali can simulate the original Margolus rule in the sense of Eq-(3-11), however, for any initial configuration

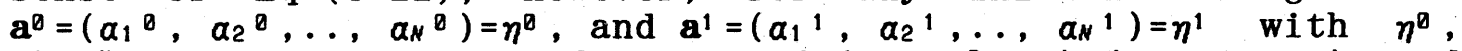
$\eta^{1} \in \Omega^{N}$ involving $111-$ suited values of $\left(x, a_{i}{ }^{\theta}, y\right)=(1,0,1)$ and $(1,1,1)$ with $x=\alpha_{i}-1^{1} \oplus \alpha_{i}{ }^{\theta}$ and $y=\alpha_{i+1}{ }^{1} \oplus \alpha_{i}{ }^{\theta}$. These ill-suited values are transmitted with the progression of time. Fig. 4 shows the time evolution of the rules with a weak self-referential property for both well-defined and ill-suited initial configurations. A well-defined initial configuration is given by a random configuration $\eta^{\otimes} \in \Omega^{N}$ and $\eta^{1}=\bar{f}\left(\eta^{\otimes}\right)$, and an 111 -suited one is given by two random configurations $\eta^{\otimes}, \eta^{1} \in \Omega^{N}$. An ill-suited configuration conflicts the local conservation law of the number of particles, and then it gives rise to that the rule cannot simulate the original Margolus rule. As far as a local universal rule is definite, the contradiction to the simulability is derived only from an initial and boundary condition and then it is maintained through the time evolution. Contrarily, in the time evolution for a well-defined initial condition no contradiction appears.

There are four rules which have no paraox or have a weak self-referential property in the rule space (3-12). Therefore we can divide the rule space into four subspaces. We here examine the behavior of a rule in $R\langle 0213\rangle$ with a finite VOP. As discussed in the above chapter, we formalize a system proceeding at a finite VOP by $\mathrm{Eq}-(2-19)$. When $\bar{\phi}: \Omega^{n+2} \rightarrow \Omega^{n}, \Omega=\{0,1\}$, is defined by the operation of $\phi: \Omega^{3} \rightarrow \Omega$ over a whole space, we here define the universality of a local rule $\phi$, which is elementary cellular automata (ECA) poposed by Wolfram (1984). Taking a finite VOP into consideration, a local rule $\phi_{c}$ which has a weak selfreferential property only has a universality over a whole space. A one-to-many type mapping appears in the form that a rule $\phi$ having no self-referential property loses its universality. Therefore, a system in ECA proceeding at a finite VOP is defined similarly as Eq-(2-19) (Gunji, 1993b) and is expressed as

$$
\begin{aligned}
& \alpha_{x} i^{t+1}=\phi\left(\alpha_{i-1} t, \alpha_{i}{ }^{t}, \alpha_{i+1}{ }^{t}\right), \\
& a_{x i}{ }^{t}=\phi^{-}\left(\alpha_{i-1}{ }^{t}, \phi_{c}\left(\alpha_{i-1}{ }^{t}, \alpha_{i}{ }^{t}, \alpha_{i+1}{ }^{t}\right), \alpha_{i+1}{ }^{t}\right),
\end{aligned}
$$

where $\phi^{-}$is chosen to satisfy that for $a_{i}{ }^{t} \in \Omega$ and a periodic boundary condition with $n$-period $\left(\alpha_{i+n^{t}}=\alpha_{i}{ }^{t}\right)$,

$$
\min _{\phi} \sum_{i}\left|\alpha_{i}{ }^{t}-\alpha_{x} i^{t}\right| \text {. }
$$

We have defined $\phi^{-}: \Omega^{3} \rightarrow \Omega$ by that if $d=\phi_{c}(a, b, c) \neq \phi(a, b, c)$ then $\phi^{-}(a, d, c)=b: \neq b$. However, we simultaneously recognize $\phi_{c}(a, b$, 
$c)=\phi(a, b, c)$ in the context of the ambiguity of the rule. Then we can also define $\phi^{-}: \Omega^{3} \rightarrow \Omega$ by that if $d=\phi_{c}(a, b, c)=\phi(a, b, c)$ then $\phi^{-}(a, d, c)=b^{\prime} \neq b$. Hence, we recognize there is no unique rule of $\phi^{-}$. Therefore we have to choose $\phi^{-}$under the condition of decreasing the metric $|\phi(\eta)-\langle\phi\rangle(\eta)|$ for $\eta \in \Omega^{n}$. The condition (316c) implies that we choose $\phi^{-}$to eliminate a paradox while there exists $(a, b, c) \in \Omega^{3}$ such that $\phi^{-}\left(a, \phi_{c}(a, b, c), c\right) \neq b$. Similarly we define a system proceeding at a finite VOP deduced from a rule in the rule space (3-12) defined as,

$$
\begin{aligned}
& \alpha_{x} ;^{t}=\phi\left(\alpha_{i-1}{ }^{t-1}, \alpha_{x} i^{t-2}, \alpha_{i+1}{ }^{t-1}\right), \\
& a_{x} i^{t+1}=\phi\left(a_{x i-1}{ }^{t}, a_{i}{ }^{t-1}, a_{x i+1}{ }^{t}\right) \text {, } \\
& a_{x} ;^{t-1}=\phi^{-}\left(b_{i-1}{ }^{t}, b_{i}{ }^{t+1}, b_{i+1}{ }^{t}\right) \text {, }
\end{aligned}
$$

for $i=1, \ldots, n$ with a periodic boundary condition, where

$$
\begin{aligned}
& b_{i}^{t+1}=\phi_{c}\left(a_{x} i^{t}, a_{i}^{t-1}, a_{x i+1}{ }^{t}\right) \\
& b_{i}^{t}=\phi_{c}\left(a_{i-1}{ }^{t-1}, a_{x} i^{t-2}, a_{i+1}{ }^{t-1}\right),
\end{aligned}
$$

and also $\phi^{-}$satisfies the condition (3-16c). This formulation gives a series of three configurations at times of $t-1, t$ and $t+1$ simulataneously. Therefore, before the next step of computational procedure for the configurations at times of $\tau-1$, $\tau, \tau+1$ where $\tau=t+3$, we define the additional procedure

$$
a_{i}{ }^{z-1}=\phi_{c}\left(b_{i-1^{t}}, b_{i}{ }^{t+1}, b_{i+1}{ }^{t}\right) .
$$

The formulation (3-17 19) embodies a one-to-many type mapping

$$
\alpha_{x} i^{t+1}=\langle\phi\rangle\left(\alpha_{x i-1}{ }^{t}, \alpha_{x} i^{t-1}, a_{x i+1}{ }^{t}\right),
$$

$t=2,3,4 \ldots$ While the time evolution of this system is puctuated by a unit configuration consisting of three time configurations, a one-to-many type mappig is expressed by $(3-20)$ whether $t \bmod 3=0,1$ or 2 . If $t \bmod 3=0, a_{x} ; t^{t-1}$ is given by the composition $\phi^{-}{ }^{\circ} \phi_{c}$. If $t \bmod 3=1, a_{x}{ }^{t+1}$ is given by the composition $\phi^{-}{ }^{\circ} \phi_{c}$. Also, if $t \bmod 3=2, a_{x i-1^{t}}$ is given by the composition $\phi^{-} \circ \phi_{c}$. We find that if $\phi=\phi_{c}$, the evolution of this system can be perfectly simulated by the weak self-referential rule in the rule space (3-12).

We estimate the behavior of a system proceeding at a finite VOP which is defined by (3-17 19) with respect to the algebraic property of $\phi$, where $\phi$ is defined in the rule space (3-12). As discused above, the rule space is divined into four sub-spaces. In each sub-space, $2^{6} / 4(=16)$ rules can be arranged by a matrix shown in Fig. 5a. The symbol wsp represents the position of a rule with a weak self-referential property. Each column represnts the value of $\left(d_{0}, d_{2}\right)$ by $(1,1),(0,1),(0,0)$ or $(1,0)$ from the upper to the lower, and each row represents the value of $\left(d_{1}\right.$, $\left.d_{3}\right)$ by $(1,0),(0,0),(0,1)$ or $(1,1)$ from the left to the right. Because $d_{1}=d_{4}, d_{3}=d_{6}$ and the value of $\left(d_{5}, d_{7}\right)$ in each sub-space is given, each lattice represents a specific rule in the rule space (3-12). For example, if the rule number of a rule with wsp is given as 22 , the corresponding rule number in a subspace is determined as shown in Fig. 5b.

That arrangement is reasonable with respect to the relationship between the behavior of a system proceeding at a finite VOP and the algebraic property of $\phi$. At first, we focus on the algebraic property shown in the columns. In the forth column $\left(d_{0}, d_{2}\right)=(1,0)$, when we denote $\left(d_{0}, d_{2}\right)$ of a rule with a weak 
self-referential property by $\left(d_{s} \theta, d_{s_{2}}\right), \quad\left(d_{0}, d_{2}\right)=\left(1-d_{s},, 1-\right.$ $d_{s}$ ). Because $\phi_{c}$ is defined as a rule with a weak selfreferential property, in a system at a finite vop deduced from a rule in the forth column of the matrix, we can uniquely determine $\phi^{-}(0,0,0)$ and $\phi^{-}(1,1,1)$ such that

$$
\begin{aligned}
& d_{\theta}=\phi\left(0, \phi^{-}\left(0, \phi_{c}(0,0,0), 0\right), 0\right) \\
& d_{2}=\phi\left(1, \phi^{-}\left(1, \phi_{c}(1,1,1), 1\right), 1\right),
\end{aligned}
$$

because $\phi^{-}$, which is defined by that if $d_{\theta}=\phi(0,0,0) \neq \phi_{c}(0,0,0)$ then $\phi^{-}(0, d \theta, 0)=0$, satisfies the condition $(3-16 \mathrm{c})$. It implies that $\langle\phi\rangle(0,0,0)=\phi(0,0,0)$ and $\langle\phi\rangle(1,1,1)=\phi(1,1,1)$ owing to the operation $\phi_{c}$, as far as $b_{i-1}{ }^{t}=\alpha_{x} i-1^{t}, \quad b_{i}{ }^{t+1}=\alpha_{x} i^{t+1}$ and

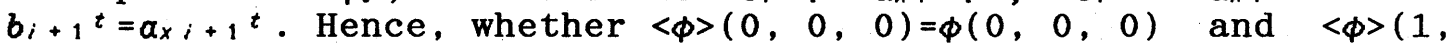
$1,1)=\phi(1,1,1)$ or not depends on the configuration at $t$ and $t \pm 1$ instants. While the value at the nearest neighboring sites are sensitive to the relationship between $d_{k}, k \neq 0,2$ and $\phi_{c}$, one can find at some instants $\langle\phi\rangle(0,0,0)=\phi(0,0,0)$ and $\langle\phi\rangle(1,1$, $1)=\phi(1,1,1)$. Then $\langle\phi\rangle(0,0,0)$ and $\langle\phi\rangle(1,1,1)$ are periodically changed due to Eq-(3-18). Hence, $\langle\phi\rangle(0,0,0)=\phi(0$, $0,0)$ and $\langle\phi\rangle(1,1,1)=\phi(1,1,1)$ are destined to be

$$
\langle\phi\rangle(0,0,0) \neq \phi(0,0,0) \text { and }\langle\phi\rangle(1,1,1) \neq \phi(1,1,1) \text {. }
$$

At a site in which there is no proceeding particle, the value of state variable constitutes a local periodic temporal sequence while that local configuration is shortened by both ends.

In general, if $d_{k} \neq d_{k}+2, k=0,1$, both triplets $(\alpha, b, c)$ corresponding to $\alpha_{j}=\phi(a, b, c), j=4 a+2 b+c, j=k$ and $k+2$ can appear in the form of $\left(a_{x} ;-1^{t}, \quad a_{x} i^{t-1}, a_{x}+1^{t}\right)$. Therefore, if $\left(d_{0}, d_{2}\right)=(0,1)$, a system behaves as well as a rule with a weak self-referential property with respect to $d_{0}$ and $d_{2}$. In this case, because not $\left(d_{0}, d_{2}\right)=(1,0)$ but $\left(d_{0}, d_{2}\right)=(0,1)$, the condition (3-22) can no longer occur.

On the other hand, when $d_{\theta}=d_{2}(e . g .=0)$, either $(0,0,0)$ or $(1,1,1)$ can appear. When one chooses $\phi^{-}$such that $\phi^{-}\left(\alpha, \phi_{c}(\alpha\right.$, $b, c), c)=b$ if $\phi_{c}(a, b, c)=\phi(a, b, c)$, one obtains that $\phi^{-}(0$, $\left.\phi_{c}(0,0,0), 0\right)=0$ because $\phi_{c}(0,0,0)=\phi(0,0,0)$ and that $\phi^{-}(1$, $\left.\phi_{c}(1,1,1), 1\right)=0$ because $\phi_{c}(1,1,1) \neq \phi(0,0,0)$. If one chooses $\Phi^{-}$such that $\phi^{-}\left(\alpha, \phi_{c}(a, b, c), c\right) \neq b$ if $\phi_{c}(\alpha, b, c)=\phi(\alpha, b, c)$, one obtains that $\phi^{-}\left(0, \phi_{c}(0,0,0), 0\right)=1$ because $\phi_{c}(0,0,0)=\phi(0$, $0,0)$ and that $\phi^{-}\left(1, \phi_{c}(1,1,1), 1\right)=1$ because $\phi_{c}(1,1,1) \neq \phi(0$, $0,0)$. While the choice of $\phi^{-}$dependent on whole configuration, one can always obtain that

$$
\phi^{-}\left(0, \phi_{c}(0,0,0), 0\right)=\phi^{-}\left(1, \phi_{c}(1,1,1), 1\right) \text {. }
$$

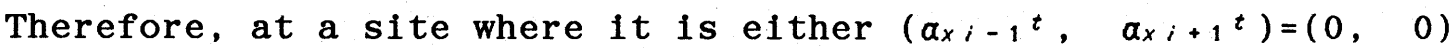
or $\left(a_{x i-1}{ }^{t}, a_{x} i^{t+1}\right)=(1,1), \alpha_{i}{ }^{t-1} \neq \alpha_{x} i^{t-1}$. We summarize that the condition $d_{k}=d_{k}+2, k=0,1$ differs from the condition $d_{k} \neq d_{k}+2$ with respect to the appearance of possible triplets and the change of value between $\alpha_{i}{ }^{-1}$ and $\alpha_{x} i^{t-1}$.

If $d_{\theta}=d_{2}=0$, the choice of $\phi^{-}$such that $\phi^{-}\left(0, \phi_{c}(0,0,0)\right.$, $0)=1$ leads to the choice of $\phi^{-}$such that $\phi^{-}\left(0, \phi_{c}(0,0,0), 0\right)=0$ soon because the former $\phi^{-}$produces a triplet $(0,0,0)$. On the other hand, if $d_{0}=d_{2}=1, \phi^{-}$such that $\phi^{-}\left(1, \phi_{c}(1,1,1), 1\right)=1$ is stably chosen under the following codition. In these cases, we have to remark the relationship between $d_{1}$ and $d_{3}$. If $d_{1} \neq d_{3}$, as well as $d_{\theta} \neq d_{2}$, both triplets $(0,0,1)$ and $(0,1,1)$ can appear with $a_{i}{ }^{t-1}=a_{x} i^{t-1}$. It implies the appearance of $(0,0,1)$ and $(0$, 
1, 1) cannot effect the appearance of $(0,0,0)$ and $(1,1,1)$. Therefore, as far as $d_{0}=d_{2}$, if $\left(d_{1}, d_{3}\right)=(1,0)$, the particle found in the original Margolus rule is also found, and the behavior of a system looks like the original Margolus rule. of corse, if $d_{\theta}=d_{2}=1,0$-particle proceeds in 1-backgrounds, and if $d_{\theta}=d_{2}=0,1$-particle proceeds in 0-backgrounds. If $\left(d_{1}, d_{3}\right)=(0$, $1)$, the relatinship between $\alpha_{x} i^{t+1}$ and $\left(\alpha_{x i-1}{ }^{t}, \alpha_{x} i^{t-1}, \alpha_{x i+1}{ }^{t}\right)$ are oscillated between the case in which they follows $\left(d_{1}\right.$, $\left.d_{3}\right)=(0,1)$ or not. Hence, one can find a perioic particle with period 3 (the value of 3 depends on the form (3-17) involving configurations at a series of three instants).

If $d_{\theta}=d_{2}$ and $d_{1}=d_{3}$, the occurence of $[(0,0,0),(1,1,1)]$ and $[(0,0,1)$ and $(0,1,1)]$ are interacted because of the change of value, $a_{i}{ }^{t-1} \neq a_{x} i^{t-1}$. Hence, at any triplets, one can find a one-to-many type mapping, and the behaviour of a system is the most complicated. As discussed later, what we are intersted in is this type of bahavior.

Finally, we can classify the bahavior of a system into five typs in the table in Fig. 5. Type 1 of a rule is found in $d_{0}=d_{2}$ and $d_{1}=d_{3}$. The characteristic behavior of this type is disorder. Type 2 is found in $d_{1} \neq d_{3}, d_{1}=d_{s} 1, d_{3}=d_{s} 3$, but not $d_{\theta} \neq d_{s} \theta, d_{2} \neq d_{s} 2$. It behaves like the original Margolus rule. Type 4 is found in $d_{1} \neq d_{3}, d_{1} \neq d_{s 1}, d_{3} \neq d_{s} 3$, but not $d_{0} \neq d_{s} 8, d_{2} \neq d_{s}$. The behavior looks like the original Margolus rule, but the proceeding particle is oscillated with periodicity 3 . Type 5 is found in $d_{\theta} \neq d_{s} \theta, d_{2} \neq d_{s} 2$. It features some periodic property. In $d_{1}=d_{3}$, but neither $d_{0}=d_{2}$ nor $\left(d_{0}, d_{2}\right)=(1,0)$, we find that any initial configuration is attracted into a stable homogeneous one. We call it type 3 . For example, $d_{1}=d_{3}=0$ in type 3 . It is trivial that if $d_{0}=d_{2}=0$ then any configuration is attracted into the configuration which consists of all 0 sites. If $d_{0} \neq d_{2}$, both triplets $(0,0,0)$ and $(1,1,1)$ can appear, while $d_{1}=d_{3}=0$ leads to the increase of a triplet $(0,0,0)$. Hence, it is also attracted into a homogeneous configuration. The classification is represented as shown in Fig. $5 b$, where a rule with a weak self-referential property is 22 . In other sub-spaces deduced from other rules with a weak selfreferential property, rule 54, 150 and 182 , the same classification is possible.

Fig. 6 shows the time evolution of a system belonging to different types and time series of the number of particles (i.e., the number of sites whose site values are $\left.1^{\prime} \mathrm{s}\right)$. The boundary condition is given by $a_{x} i^{t}=a_{x i+n^{t}}$ under the system size of $n$. Initial conditions $\left(\alpha_{x} i^{8}, a_{x} i^{1}\right), i=1, \ldots, n$ are given to be welldefined with respect to the original Margolus rule. It implies that a rule with a weak self-referential property can behave similarly as the origianl Margolus rule. In this simulation, we find that a system proceeding at a finite VOP proceeds to satisfy a logical consistency and that satisfying the logical consistency is replaced by the conservation of the number of particles. Type 2 and 4 rules soon reach the configuration in which the number of particles can be conserved. In type 3 rules the number of particles is conserved either with 0 or $n$. In type 5 , the number of particles is oscillated, and it implies that there exists some conservative law. All of rules of these types can realize conervation laws. The effort to realize the conservation of the number of particles can be more or less achieved. If one calculates the time evolution of $\phi$ under the assumption of an infinite VOP, the patterns of evolution differ from those shown in Fig. 6. Fig. 7 shows the time evolution of $\phi$ is prepared by the matrix shown in Fig. 5. Note that one cannot classify the 
pattern by the algebraic properties discussed above.

Fig. 8a represents $\sigma^{2}$-of the number of particles in 2000 instants for ecah rule. Four matrices sorrespond to four subspaces in the rule space. Each matrix is divided into 16 box elements, and the length of a black bar in the horizontal axis in each box represents $\sigma^{2}$. The (2nd column, 2nd row) box in each matrix corresponds to a rule with a weak self-referential property. The arrangement of rules in each matrix follows Fig. 5, where the rule with a weak self-referntial property in the upper left, upper right, lower left and lower right matrix is 22, 150 , 54 and 182. It is clear that there exists a conservative law except for type 1 and 5 . Fig. $8 \mathrm{~b}$ represents a mean value of the Hamming distance defined by (3-16c) in 2000 instants of evolution, where it is normalized by the system size $n$. The presentaion of this diagram is similarto the one in Fig. 8a. It shows that there is no paradox ( $1 . e$. , the Hamming distance is 0 ) if the number of particles is conserved.

Fig. 9 represents mean values in the time series of the spatial metric entropy defined as

$$
S(X)=\stackrel{2}{k}_{i}^{*} p_{i} \log _{2} p_{i}
$$

where $X$ represents the box size. Fig. $9 a$ and $b$ show the mean values of $S(2)$ and $S(3)$ respectively. The arrangemnt of these matrices and boxes are similar to the ones in Fig. 8. The rule with a weak self-referential property has the value of 1 whether $X=2$ or 3 . That random initial configurations are conserved in that rule is reasonable because it conserves any configuration once it is given. It has a universality in the terminology of computation theory. On the other hand, even in type 1 rules one finds a high value of $S(X)$. It has no conservation law and it implies that it loses a universality. The algebraic property of the rule $\phi$ in type 1 greatly differs from $\phi_{c}$. That implies that type 1 rules are specialized and more irreversible. Hence, it may realize an abnormal computation of which both the universality and high efficiency are achieved to some extent, while there is a trade-off in the programmable systems. It will be discussed in the next chapter.

Type 1 rules cannot achieve the conservation of the number of particles. Although this type of a rule functions so as to achieve the conservation law, such efforts is destined to end up in vain. The motion for achieving the conservation law paradoxically leads to a drastic change in the number of particles and then it again elicits the motion for achieving the similar conservation of the number of paritcles. It is perpetually repeated and is disequilibrated. As a result, complex patterns are originated with the elapse of time. The difference between type 1 rules and those of other types are now regarded as the difference between disequilibrated and near equilibrated systems. We call the behavior of the type 1 rules disequilibration. In the next section, we examine the properties of disequilibration in the context of a language.

\section{3-3. language not as a rule but as a game}

A system proceeding at a finite VOP results in a disequilibration more or less. What is intriguring to the system proceeding at a finite VOP is its characteristic of bringing. forth a unity. In any computers in which programmable statements 
can be used, the assumption of an infinite VOP can be replaced by prohibiting the receipt of another information in the midst of computation. If this prohibition fails, the computer is destined to face dead-lock. We are not interseted in such systems. On the other hand, in any biological system including our language community, one can find that a language game is played as proceeding at a finite VOP. It implies that a game entailing a unified system proceeds while there exists no rule embodying such a game. In general, a language game is believed to be explained by a specific rule, and then one intends to find a specific rule. However, the finiteness of VOP lets us conclude that all what one can do in terms of finding a rule is to invent a rule by which a specific game can approximately explained. The relationship between a language game and its rule is reverse(Gunji, 1993a).

Any communication and interaction must have a logical consistency in terms of measurement and description of which an observer must assume an infinite VOP. As discussed above, in computers, once the logical consistency fails as facing a deadlocking, it cannot be recovered any more. On the other hand, in biological systems, even if a specific rule is broken another specific rule may look to originate instead. One can regard this aspect as if there was an aim to maintain a logical consistency. We can sketch a system prceeding at a finitde VOP as a motion to maintain logical consistency in spite of the transformation of the language, if we measure the system. We can here distinguish a system of which the aim to maintain the logical consistency can be achieved from a system of which it cannot be. Finally we define the former by equilibration and the latter by disequilibration. Of course, the former case is achieved only if a system proceeds at an infinite VOP, then in physical phenomena such a distinction is arbitrary or is possible with respect to the approximation. However, because VOP of non-biological system is much larger than that of a biological system, we can distinguish equilibration from disequilibration.

It is clear that communications related to our language, intelligence and autonomy proceeds at a finite VOP. As discussed above, the resulting desription approximated by the assumption of an infinite VOP is destined to be a paradox. Therefore our formulation such that a paradox is regarded as a motion essentially differs from the approximated form with an infinite VOP. That difference is clear in understanding what a language is. We here call all communicative phenomena langauge games. If one assumes an infinite VOP, a specific langauge game is reduced to a speicific rule, and one intends to examine the origin of the rule. The research in the framework of Artificial Life shows one of the examples in this trend(e.g., Werner \& Dyer, 1991). Various different rules are originated at random, and they are continued to be selected by a specific fitness function corresponding to a specific environment untill a stable rule is achieved. Hence, the transformation of a rule is reduced by another transformation of environments. Once a rule is chosen, it is stable as far as it is not perturbed. A language is regarded as a form of equilibration. In our framework, a langauge game is designated as a disequilibration. One cannot find a unique rule by which a language game can be deduced in principle. In other words, one can find a global logic which cannot be reduced to a local rule in disequilibration. Type 1 rules in our system embodies that aspect. As shown in Fig. 6, the behavior of type 1 rules looks like the disorder and there is no specific pattern. However, a specific initial condition leads to a speicific pattern 
propagating regularly (Fig. 10). Given a particle initially, it propagtes expanding its width and constitutes a gasket-wave. As well as a motion of a particle in the original Margolus rule, a gasket-wave propagates toward either the right or the left direction. This gasket wave has a specific property with respect to two points, which features disequilibration.

The first point is that a gasket-wave has a property of a particle while it propagates like wave. Fig. 10 shows that two gasket waves go through after the collision, which is a character of solitons. The horizontal black bar at each instant(Fig. 10), which is accopanied with the evolution of a gasket-wave, represents the number of particles at each instant. Comparing the evolution of one gasket-wave with that of two, one can find that twice the number of particles in one gasket-wave equalsthat in two gasket-waves. In spite of their collision, the gasket wave propagates as if there were no collisions. The gasket-wave basically consists of a local check pattern. Therefore, as far as the two waves are not located in the same position, the number of particles cannot be decreased by collisions. Indeed, there is no such case that two waves propagating in two opposite directions collide in the manner that these particles constituting different wave-gaskets are located in the same positions.

Fig. 11 shows the relationship between the distance of two gasket-waves and the direction of their propagation. If the two gasket-waves are separated over $2 n$ sites (i.e., the seed of the first wave is located at the $i$-th site and that of the second wave is at the $(i+2 n)-$ th site), they propagate in the same direction. They cannot collide as propagating in the opposite direction with each other, while they constitute a single emergent wave as interfering with each other. On the other hand, if the two waves are separated over $2 n+1$ sites, they propagate in the opposite directions. In this case, the two waves collide. At the moment of collision, one particle in the one gasket-wave occupies a hole in the check pattern of the other gasket-wave. Therefore, each of the two waves propagating in the opposite directions can pass through the other without interaction. Note that $n>1$. Two seeds separated over between two sites yields only one gasket-wave because one seed is cancelled by the other. This cancellation at the instant $t$ depends on the value of the state at the instant $t-1$ and $t-2$. Any pair of gasket-waves approaching with each other are either interfered or superimposed. This feature is important in the global computation.

The second point is that a gasket-wave is not reducible to a specific local rule. In our framework of a system proceeding at a finite VOP, the procedure $(3-17,18)$ yields a formal device for prescribing the aspect that a local rule $\phi$ in a given rule space is operated, while requiring a finite time interval. Therefore, the behavior of the system is foundamentaly prescribed by the algebraic property of a map $\phi$. Despite this, it also shows that under the procedure $(3-17,18)$, the VOP of the system is expressed by the algebraic property of $\phi$. If $\phi$ has a weak selfreferential property, the behavior of the system with the procedure $(3-17,18)$ is the same as that of $\phi$ without that procedure. It implies that the system proceeds at an infinite VOP. On the other hand, type 1 rules such that for any $(a, c) \equiv \Omega^{2}$, $\phi(x, 0, y)=\phi(x, 1, y)$ where $x=a \oplus b, y=b \oplus c$ are most far away from the rule with a weak self-referential property because the latter is defined in such a manner that for any $(\alpha, c) \in \Omega^{2}, \phi(x, 0$, $y) \neq \phi(x, 1, y)$ where $x=\alpha \oplus b, y=b \oplus c$. It implies that type 1 rules under the procedure $(3-17,18)$ proceeds at the smallest VOP in 
the rule space, which entails that the system realizes perpetual disequilibration. In other words, type 1 rules under the procedure $(3-17,18)$ realize a system proceeding at a finite VOP while the inter-cellular transition rule looks as if defined in the form of $\phi: \Omega^{3} \rightarrow \Omega, \alpha_{i}{ }^{t+1}=\phi\left(\alpha_{i-1}{ }^{t}, \alpha_{i}{ }^{t-1}, \alpha_{i+1}{ }^{t}\right)$. In this context, if one sticks to describing the evolution of type 1 rules in the form of $\phi: \Omega^{3} \rightarrow \Omega$, it is destined to be of a one-tomany type mapping. For example, in a gasket-wave, one finds both $\phi(1,1,1)=0$ and $\phi(1,1,1)=1$.

With respect to those two points, one can find that a global pattern appears while it is by no means reducible to local rules. It is, however, noted that the global or spatial distribution of a particle is reducible to a local or temporal probability of the existence of a particle in equilibrium because there is no deterministic rule. It is suggested that a stochastic process may imply that VOP is zero, and that if VOP is zero, then one can find a random process featuring probabilities instead of a deterministic rule. On the other hand, if VOP is infinite, the global pattern is perfectly reducible to a local rule because the globality is tautologically prescribed by a local rule. In the sepctrum of VOP except for the extreme-case members(VOP=0 or $\infty$ ), one comes to face the evolution which cannot be estimated in terms of either detriminstic or stochastic scheme. Infinite or zero VOP features a ideal system in which each of locality and globality are reducible to the other on the one hand. Finite VOP suggests an idea of a hierarchical structure because each of local and global features, which cannot be reducible to the other, generate and are generated by disequilibration.

The global pattern resulting from disequilibration suggests the model of a langauge game which cannot be reduced to a rule of a language. Because each gasket-wave behaves independently and is regarded as a particle, one can control a stream of a gasketwave. In ballistic computations (Toffoli \& Margolus, 1987), a collision of two particles is utilized as AND or NOT gate in a two dimensional lattice. One can compute any statements in twovalued logic by a ballistic computation where one regards the presence of a particle as valued 1 and the absence as valued 0 . For example, a particle that can collide with a given particle and shifts its orbit can be used as a NOT gate. If an input value is 1 implying the presence of a particle, it collides another particle which is prepared as a NOT gate and the orbit is shifted. At this time, if one waits for a particle on the site where it is expected that a NOT-gate-particle presents, one cannot find a particle and obtains a value of output as 0 . On the other hand, if the value of input is 0 implying the absence of the particle, one waiting for a NOT-gate particle at the same site can find it and obtains its value 1. Therefore, one can utilize a collision as a NOT gate.

Two gasket-waves can be superimposed, then the collision of the two gasket-waves can be utilized as an OR gate. If one represents the presence of a gasket-wave as the value 1 , a pair of input values $(A, B)=(0,1),(1,0)$ and $(1,1)$ are realized by the presence of one or two gasket-waves and it leads to an output value 1 on the one hand, and $(A, B)=(0,0)$ leads to 0 on the other. However, one may find that it is not practical because the presence of a particle have two different directions. If the output of the operation of $O R$ is successively utilized, it is more useful that the direction of a gasket-wave may be controlled. When one introduces the cancelation of a particle such that if there is at least one particle at instant $\tau$ then it 
will be set at a specific site at instant $\tau+1$, one can control the direction of the suceeding gasket-wave (Fig. 12).

Also, we can construct a NOT gate by using a specific boundary condition, called a wall. The wall consists of the primary-wall prescribed by $\left(a_{x} s^{\tau}, a_{x} s^{\tau+1}, a_{x} s^{\tau+2} \ldots, a_{x s^{\tau+m}}\right)$ such that for the state of $a_{x} s^{r+k}, k=0,1, \ldots, m$, the state of the neighboring site is defined by $\alpha_{x} s+1^{\tau+k}=\alpha_{x}-1^{\tau+k}$, and the

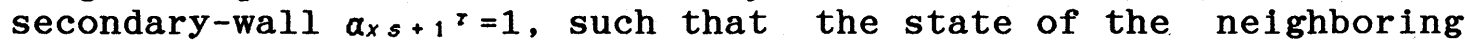
site for the state $\alpha_{x} j^{r k}, j=s+1, s+2, \ldots, N$, is defined by $\left(a_{x} j-\right.$ $\left.1^{\tau+k}, \alpha_{x /+1^{\tau+k}}\right)$. Therefore, if a gasket-wave proceeds from the left side area ( $i . e . i<s)$ and collides with the wall, it is reflected and proceeds in the reverse direction. If there is no gasket-wave approaching the wall from the left side, the secondary-wall plays a role of yielding a seed of a gasket-wave to be emerged. As far as one sets the timing $\tau$ at which a gasketwave reaches the wall if it exists, the seed in the secondarywall is cancelled only if the gasket-wave reaches the wall. Finally, given a gasket wave approaching the wall which implies the input value 1 , one waiting for a gasket-wave at the opposite side of the wall obtains no gasket-wave, which implies the output value 0 on the one hand. If there is no gasket wave (i.e. input value is 0 ), one obtains the output value 1 which results from the seed in the secondary-wall. Hence, we can utilize the wall as a NOT gate.

Because we construct both NOT and OR gate in the pattern consistsing of a gasket-wave, other operations of Boolean logic in this system also follow the suit. The gate AND is made of both NOT and $O R$ gates, because $A \wedge B=>((>A) \vee(>B))$. The gate IMPLICATION is also possible because $A \rightarrow B=(>A) \vee B$. Finally, all operations in Boolean logic can be expressed by utilizing the interaction of gasket-waves, because any other operations can be expressed only by NOT and/or OR. In other words, one can construct Boolean logic in a system of type 1 rules, and it is proven to be capable of supporting universal computations. However, it is remarkable that we introduce some interactive device between a system and a user like the wall. In spite of that introduction, is it possible to say that the very system is capable of universal computations in its own right? To answer this question we have to reestimate the idea of universality and/or computation.

We here estimate the relationship between universality and efficinecy. When one describes reversible and irreversible systems in the state space, the reversible system is described as a bijective map on the one hand and the irreversible system as a many to one type mapping. Comparing the reversible system to the universal computation, one understands that the universal computation trades off its high efficeincy, which is called the trade-off principle (Conrad, 1987). While any configuration can be reached from a specific initial configuration and the resulting specific configuration is regarded as an output, it takes a long time to compute the output as letting it be dependent on the initial configuration. One who uses such a universal computer has to wait for a specific configuration and has to grasp it successfully. If one misses a configuration in the form of an output, he has to wait twice the time. On the other hand, initial states in a highly irreversible system are expected to be attracted into a speicific stable fixed point. Hence, computation is highly effective as for a specific configuration, though it is not available for a universal computation.

In the previous works (Gunji \& Konno, 1991; Konno \& Gunji, 
1991; Nakamura \& Gunj1, 1993), we classify the rules of ECA into two types, reversible and irreversible, while the rules of ECA are essentially irreversible because of the form $\phi: \Omega^{3} \rightarrow \Omega$. Reversible rules in ECA implies that for any $(a, \phi(\alpha, b, c), c)$ there exists a map $\phi^{-}: \Omega^{3} \rightarrow \Omega$ such that $b=\phi^{-}(a, \phi(a, b, c), c)$. Ito \& Gunji (1992) and Gunji \& Ito (1992) extended the idea of reversibility in cellular automata as featuring the interaction with the next-nearest neighboring sites, and confirmed that reversible system shows the feature of the edge of chaos proposed by Kaufmann \& Johnsen (1991). Especially, the game of life (Gardner, 1970) is a unique reversible rule in the rule space $\left\{\phi: \Omega^{5} \rightarrow \Omega\right\}$ in two-dimensional lattice. If one assigns a speicific boundary condition, initial configuration can be obtained from the final configuration computed by the game of life. Hence, the game of life is located in the edge of a universal system, and it has the features of both of reversible and irreversible system. In fact, the game of life can construct a Boolean logic by the speicific configuration called glider gun (Berlekamp et al., 1982). In this context, the universal computation is a character of reversible system.

Langton (1991a, b) emphasized that a system featuring the edge of chaos is capable of a universal computation in spite of its irreversibility, and that the system exhibits a foundamental feature of the phenomenon of life. His argument is that biological communications related to the intelligence are at the edge of chaos. This idea is related to the computation thoery in which computation is necessarily irreversible, because extracting an output is realized by halting the computer. In this context, chaotic dynamics embodies indeterminacy because it involves the haliting problem of which there is no algorithm to determine whether a computer halts or not. The indeteminacy is originated from that the domain of dynamics, $V$, consists of a Cantor set and accumulating points (Devaney, 1986). The aspect that at the edge of chaos it is capable of computation for removing the indeteminacy may be regarded as something suitable for intelligence. However, the ability of computation exhibited in organisms may not be as the same as that in a system featuring the edge of chaos. There are two reasons. First, as discussed above, it is reasonble that a system featuring the edge of reversibility is capable of a universal computation and constructs a Boolean logic. The universal computation results from reversibility, and not from the edge of chaos, while the system at the edge of chaos can be reversible in the above sense. The original Margolus rule and type 2 rules in our Margolus system can also construct a Boolean logic in the context of ballistic computaion (Toffoli \& Margolus, 1987), though it is not at the edge of chaos. Also, the edge of reversibility is expected to show the intermediate pattern in the spectrum covering from order through disorder.

The second reason is more serious. The computation in the game of life at the edge of chaos is expressed in a programmable system, while biological systems are unprogrammable due to the finiteness of VOP. Therefore, it is possible that resulting universality cannot be reduced to a local rule which is programmable. If biological computations can be expressed in the form of a formal programming, they cannot be beyond the trade-off principle. However, if unprogrammable in principle, all programmale computations one can find in biological behaviors are destined to be restricted as anapproximation. High efficient computation is possible, while universality can only be a matter 
of approximation.

Type 1 rules is capable of a universal computation, while one has to introduce the cancellation device and the wall for a NOT gate. It shows that the universality may not be perfect on the one hand, but also shows the essential aspect of computation. If one says that a system shows a universal computation, one will be to forget that no computation is possible without its user. One can use a computer, not because the computatin theory is defined as being separated from its user or from the measurement procedure of its user, but because the user measures the resulting output and the computer is fabricated and constructed so as to yield the output which is measurebale by the user. The measurement and fabriacation of the computer brings forth the process of self-reference, and accordingly, one cannot prescribe the measurability as a metter of principle (Gunji \& Nakamura, 1993). In other words, computation is well-defined only if semantics is defined and if the semantics is replaced by metrics in the state sapce. Therefore, it always implies the existence of an observer or user who can observe the system at an infinite VOP.

Even in reversible and programmable system, the ability of computation is estimated by an observer who observes the global space. In the game of life, for given values of the inputs for a specific timing and location of a glider, one has to know both the specific timing and the location of the resulting glider colliding with the glider of the gate which determines the value of the output (Berlekamp, 1982). Also in a ballistic computation, one has to know the expected orbit of a particle (Toffoli \& Margolus, 1987). Although prescribing a specific timing and location is just a matter of measurement, we have here confirmed that the measurement is not distinguished from a system. Therefore, we cannot distinguish measurement and/or prescribing the resulting configuration as an output from the device of the wall in the computation in a system of type 1 rules in a Margolus system. Note that as far as one Ignores the measurement involved in computation which entails separation of the computer from the measurement, one can regard the computation theory as a real objective and can define both universality and efficiency in programming.

Therefore, we emphasize that the trade-off principle is right in the programmable system in which we believe that probrammability is proved while a true criterion of programmability is not attainable. The trade-off principle is the end member in the spectrum of computation which involves an unprogrammbale computation (Conrad, 1987). One cannot separate between boundary conditions and the process of measurement. This inseparability applies even to $a$ a specific interface. with boundary conditions. It is of course possible that one can define the criterion of a programmable system and define a universality such that the observer does not interact with the system except at the time of measuring the output. That criterion is recognized as being objective in general, but is still arbitrary. We can extend the Idea of computation in the manner that the definition of universality is extended so that a specific interaction between the system and the user can be allowed. Consequently one can find that a universal but high efficient system becomes possible while the criterion of efficiency and universality has already been modified.

Does it mean that any programmable system that is capable of high efficient computation can be regarded as a universal 
processor? The answer would be yes if it is proved that any interaction is possible in any system or if one can move a particle without moving other particles. The answer is no, however, because we have extended the concept of computation so as to include computations in unprogrammable systems. We admit that a system proceeding at a finite VOP can realize a computation. Therefore, answering yes to that question is destined to fail. If one defines an arbitrary criterion of universality only in the programmable system, the trade-off principle can be modified but remains isomorphic to the original trade-off principle. A highly efficient but universal computation found in a system proceeding at a finite VOP is outside from the category of programmable computation. We cannot evaluate it in terms of the trade-off principle in programmable systems.

In fact, universal computation found in a system of type 1 rules has a high efficiency. To see this, one can compare it to be a ballistic computation. Imagine a value of 1 of a ballistic computation in the orginal Margolus CA consisting of n-lattices and with a periodic boundary condition. One can obtain a value of 1 when one finds a particle proceeding along a specific orbit in any site of one-dimensional space. Therefore, given a proceeding particle, the number of possible configurations yielding a value of 1 is $n$. Next, imagine the same situation in a system of type 1 rules. A value of 1 is obtained when one finds a gasket-wave. Because the gasket-wave propagates as expanding and changing its own pattern, the number of possible cofigurations which is observed by its user is $2^{n}-1>n$. It implies that one can choose a site in which one measures a value of a particle more roughly in a system of type 1 rules. If one loses sight of a gasket-wave at a spcific site, one does not have to waste the time. If one takes an uncertainty in measurement into consideration, that system will turn out to have a high efficinecy. It is reasonable because the system proceeding at a finite VOP is always accompanied by an uncertainty.

We emphasize a biological computation resulting from disequilibration. When one sticks to a programmable computation, a biological system would have to realize "computation" while it finds itself whithin the realm of the halting problem. In other words, we can find an occurence of computation in biologically disequilibrated proccesses and also find information generation. It looks as if we would interpret an arbitrary biological process as a computation. This interpretation is, however, not correct. No computations including programmable ones can be possible without the observer or the involvement of measurement. Computations in disequilibration require user's intervening with the computer more often than programmable computations do. As a result, information generation is a consequence of thetrading off in programmablity.

\section{Conclusion}

The idea of a system proceeding at a finite VOP leads to disequilibration process. If one attempts to describe the process, a self-referential form or a paradox will follow because the description of interaction involves the metric featuring the wholeness that assumes the idea of a system proceeding at a infinite VOP. With respect to the indeterminacy in the self-reference, chaotic dynamics can be a model for such a disequilibrated process. However, we find that the identification of a chaotic attractor with a one-to-many mapping (i.e. the 
assimilation of a bundle of trajectries to a single trajectory) is a paradox in its own right. It leads the asepct that one has to admit the meaning(value)-change as time proceeds, if one claims the identification of the meaning a priori. It does not imply that $b^{\prime}=f(a)$ where $f$ needs a finite time interval from $t$ to $t+\Delta t$ instants, but that after the identification of $b^{\prime}=f(a)$ one can find $\alpha^{\prime} \neq \alpha$ as the value of the state at the $t$-th instant. This is the idenfification occured a posteriori.

To demonstrate what the identification a posteriori is all about, we proposed the model called a self-referential system that consists of both forward-and backward-time dynamics, and replace the identification of local meanings consistent with the global meaning by conservation law of the number of particles. Corresponding to the algebraic property of the forward-dynamics in the self-referential system indicating the level at which the consistency is satisfied, the behaviors of the self-referential system in the rule space called the Margolus rule are classified into five types. The self-referential system positioned most far away from the conservative system exhibits a disequilibration, in which the system paradoxically realizes non-conservation due to the conservation law. Even if there is a logic to conserve the number of particles, it cannot be realized because the semantical value of the very logic propagates at a finite velocity. Therefore, the disequilibration process embodies an indeterminacy as a matter of principle, and then it cannot be regarded as a computation in the standard framework of programmable computation.

We can, however, find a computation in disequilibration in the sense of trading-off programmable computation. Though it may look as if the computation results from an arbitrary interpretation of the observer participating in the context of "finding", it rather shows that no computation proceeds without observation and that a computer cannot be separated from its user. This aspect is easy to forget. Even in the programmable computation, the computer is constructed so as to yield an output that can be used as an input to subsequent computation, while one often misunderstands that there would exist a reality yielding the foundation of the usage. That misunderstanding takes one from "to use" to "to be able to use", and leads to the idea of control.

Biological computation gives rise to the aspect that the measurement which is hidden in the programmable computation explicitly comes to the surface. Hence, we as users of a computer positively intervene with the computational process. In our selfrefererential system in the Margolus rule space, one can find a mode of computation in a system featuring disequilibration through introducing a specific boundary condition. The resulting computation or the computation found in this case, however, is a Boolean logic consisting of a gasket-wave pattern. We emphasize the following two points. First, biological computation implies neither the computation theory differing from that for programmable computation nor a form of an inconsistent language. It implies a consistent langauge as well as a programmable computation theory. Second, the user of a biological processor has to positively intervene with the processor, while the results of intervention must be uncontrollable. The intervention is always accompanied by an indeterminacy. When we wish to fabricate a biological processor in the future, it will not be expected that the processor may arise independently of heuristic ideas on the part of users. Rather, the user manages how to use the 
processor with the much greater effort than to use the processor. to extract only its output. The situation is similar to a natural language. Undestanding disequilibration gives rise to understanding both the phenomenon of $11 \mathrm{fe}$ and a natural language that cannot be reduced to the rule of a specific languague. Our self-referential system can serve as a model for understanding disequilibration.

Acknowledgement: We specially thank to Matsuno; K. for numerous suggestions.

\section{References}

Berlekamp, E., Conway J. \& Guy, R., 1982, Winning Ways for Your Mathematical Plays. Academic Press, New York.

Conrad, M., 1985, On design principles for a molecular computer. Communications of $A C M$ 28:464-480.

Conrad, M.; 1987, The price of programmablity, in R. Herken (Ed.), The Universal Turing Machine: A half-Century Survey, Oxford Univ. Press.

Crutchfield, J.P. \& Kaneko, K., 1987, Phenomenology of spatiotemporal chaos. in Crutchfield, J.P. \& Kaneko, K . (eds.), Directions in Chaos, World Sc. Co., Singapore

Devaney, R.L., 1986, An Introduction to Chaotic Dynamics. Benjamin/Cunnings Pub.Co., New York.

Freishaker, G.R., 1988, Autopoiesis: the status of its system logic. Biosystems 22: 37-49.

Gardner, M., 1970, Mathematical games. Sci. Am. 223(4):120-124.

Gunji, P-Y., 1993a, Form of life: Unprogrammability consitutes the outside of a system and its auotnomy. Appl.Math.Comput (in press).

Gunji, P-Y., 1993b, Autonomic life as the proof of incompleteness and Lawvere's theorem of a fixed point (submitted to Appl.Math.Comput.) .

Gunji, Y. \&.Konno, N., 1991, Artificial life with autonomously emerging boundaries. Appl. Math. Comput. 43:271-298.

Gunji, Y. \& Nakamura, T., 1991, Time reverse automata patterns generated by Spencer-Brown's modulator: Invertibility based on autopoiesis. Biosystems 25:151-177.

Gunji, P-Y. \& Nakamura, T., 1993, Measurement problem in biology: a fixed point dericed from a finite velocity of observation propagtion (submitted to Systems Research).

Gunji, P-Y. \& Ito, K., 1992, Evolutionary dynamics of complex systems: self-organization toward criticality at the border between order and chaos. In S. Ushiki (Ed.), Structure and Bifurcations of dynamical systems, pp. 59-81, World. Sc.

Ito, K. \& Gunji, P-Y., 1992, Self-organization toward criticality in the game of 1 ife. Biosystems 26:135-138.

Kaneko, K., 1989, Simmulating physics with coupled map lattices. in Kawakami, K. (ed.), Formation, Dynamics, and statistics of Patterns. World Sc. Co., Singapore.

Kauffman, S. A. \& S. Johnsen, 1991, Coevolution to the edge of chaos: Coupled fitness landscapes, poised staets and coevolutionary avalanches. J.theor.Biol. 149:467-505.

Kirby, K.G. \& Conrad, M., 1986, Intraneuronal dynamics as a substrate for evolutionary learning. Physica 22D:205-215.

Konno, N. \& Gunji, Y., 1991, Mathematical construction of an autonomous artificial life. Appl.Math.Comput. 46:33-58 .

Langton, C. G., 1991a, Computation at the edge of chaos. Physica 22D : $12-37$. 
Langton, C. G., 1991b, Life at the edge of chaos, in C.G. Langton (Ed.), Artificial Life II, pp.41-91, Addison-Wesley.

Margolus, N., 1982, Physics-like models of computation. Physica 10D: $81-95$.

Matsumoto, K., 1984, Noise-induced order II. J.Stat.Phys. 34:111126 .

Matsumoto, K. \& Tsuda, I., 1983, Noise-induced order I. J.Stat.Phys. 31:87-106.

Matsuno, K., 1989, Protobiology: Physical Basis of Biology. CRC press, Boca Raton, FL.

Maturanra, H.R. \& Varela, F.J., 1980, Autopiesis and CognitionThe Realization of the Living. D. Reidel Pub.Co.

Nakamura, T. \& Gunji, P-Y., 1993, Algebraic properties of backward dynamics(submitted to Appl.Math.Comput.).

Nicolis, J., 1991, Chaos and information processing. World Sc. Co. Singapore.

Toffoli, T. \& Margolus, N., 1987, Cellular Automata Machines. MIT Press, Cambridge MA.

Varela, F.J., 1979, Principles of Biological Autonomy. NorthHolland.

Werner, G.M. \& Dyer, M.G., 1991, Evolution of communication in Artificial Organisms, in C.G. Langton (Ed.), Artificial Life II, pp.659-687, Addison-Wesley. 

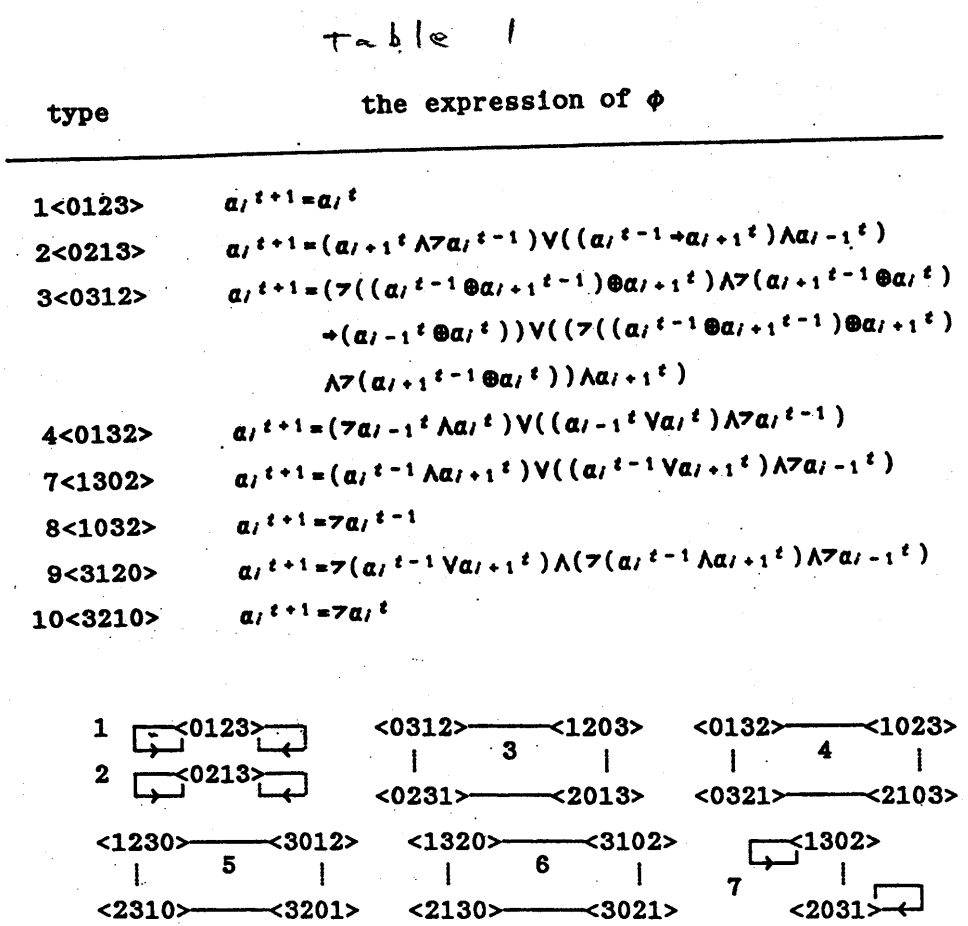

$8 \square \square^{1032>-<2301>\square 9}{ }^{9} \square^{<3120>\square}{ }^{10} \square^{3} 3210>\square$ Fig. 3
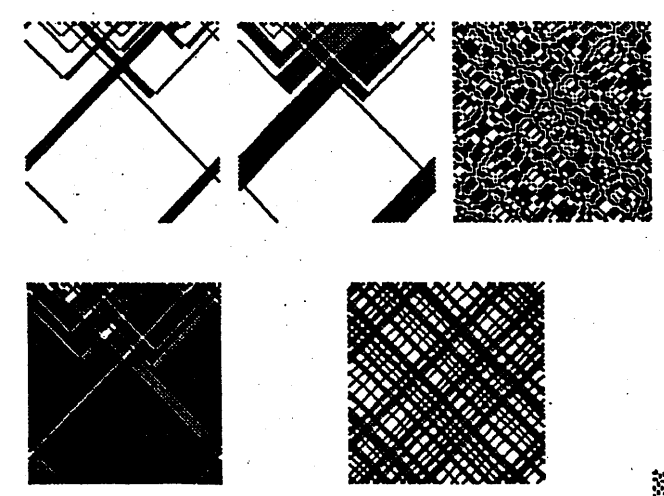

Fig. 4

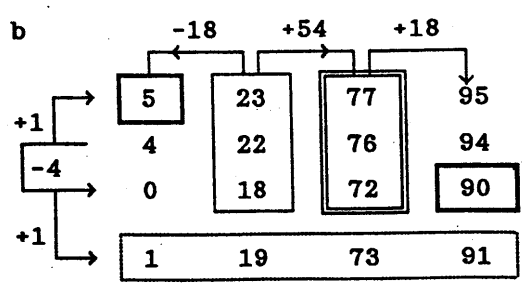

$F: 8=b$

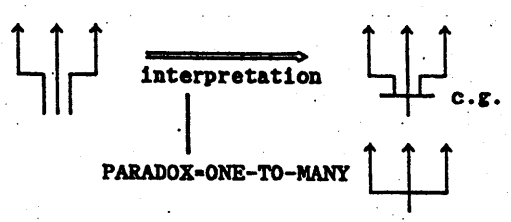

$F: q . \quad 1$
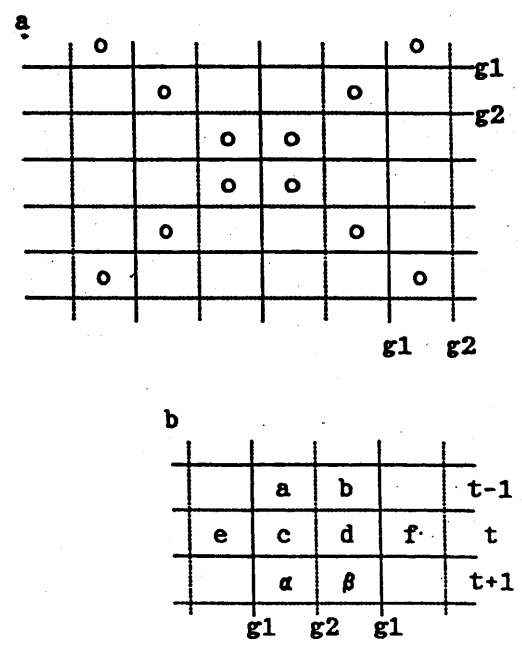

$F: g .2$ a
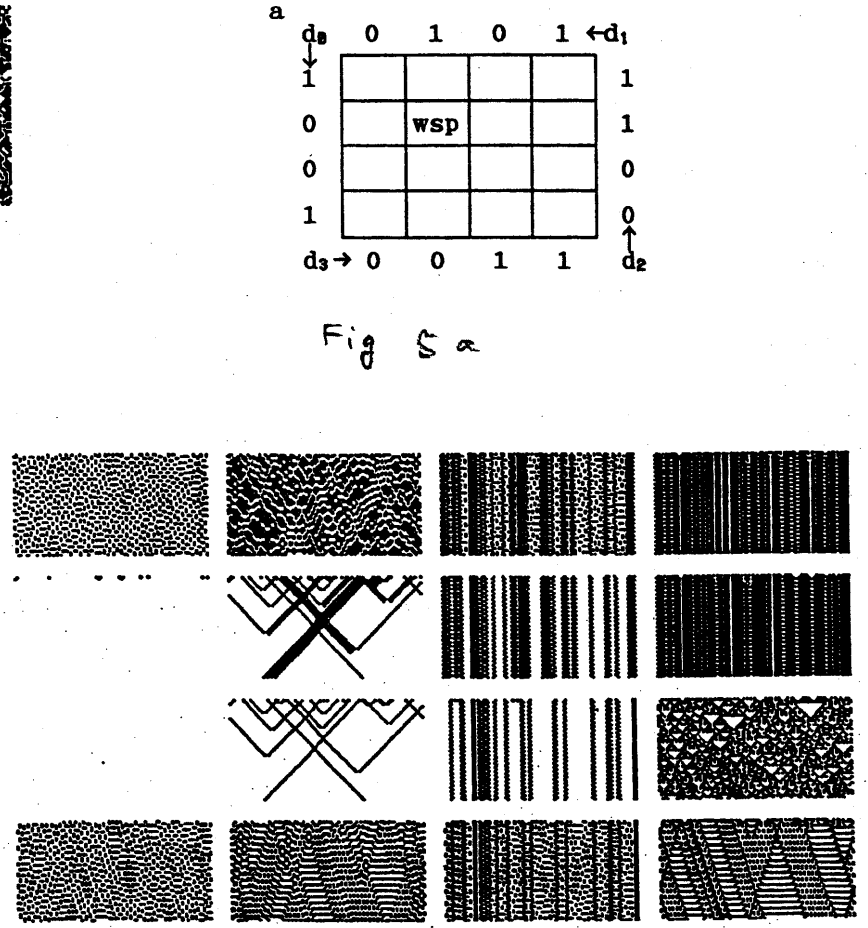

$F: 2.7$ 

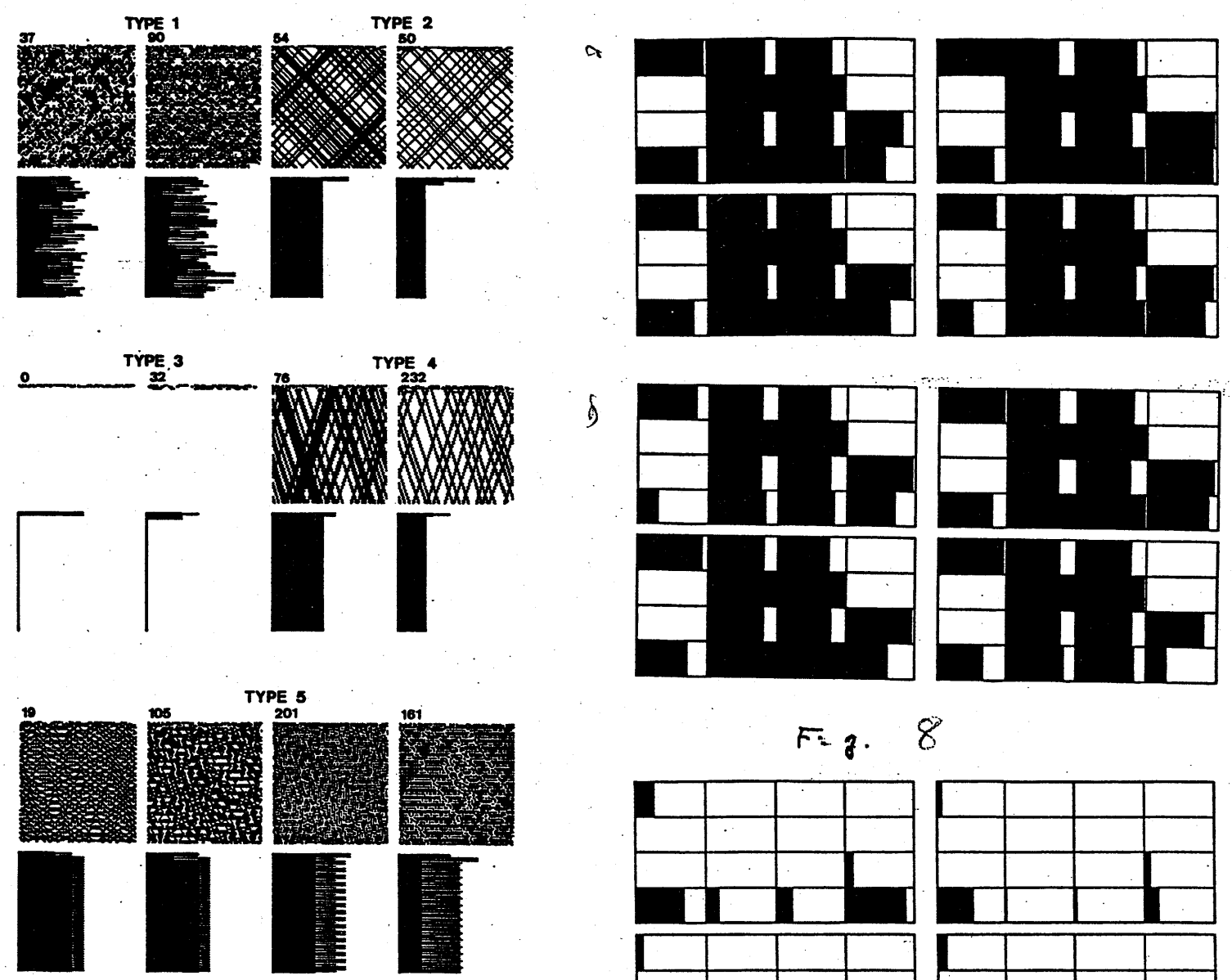

F.g. 8
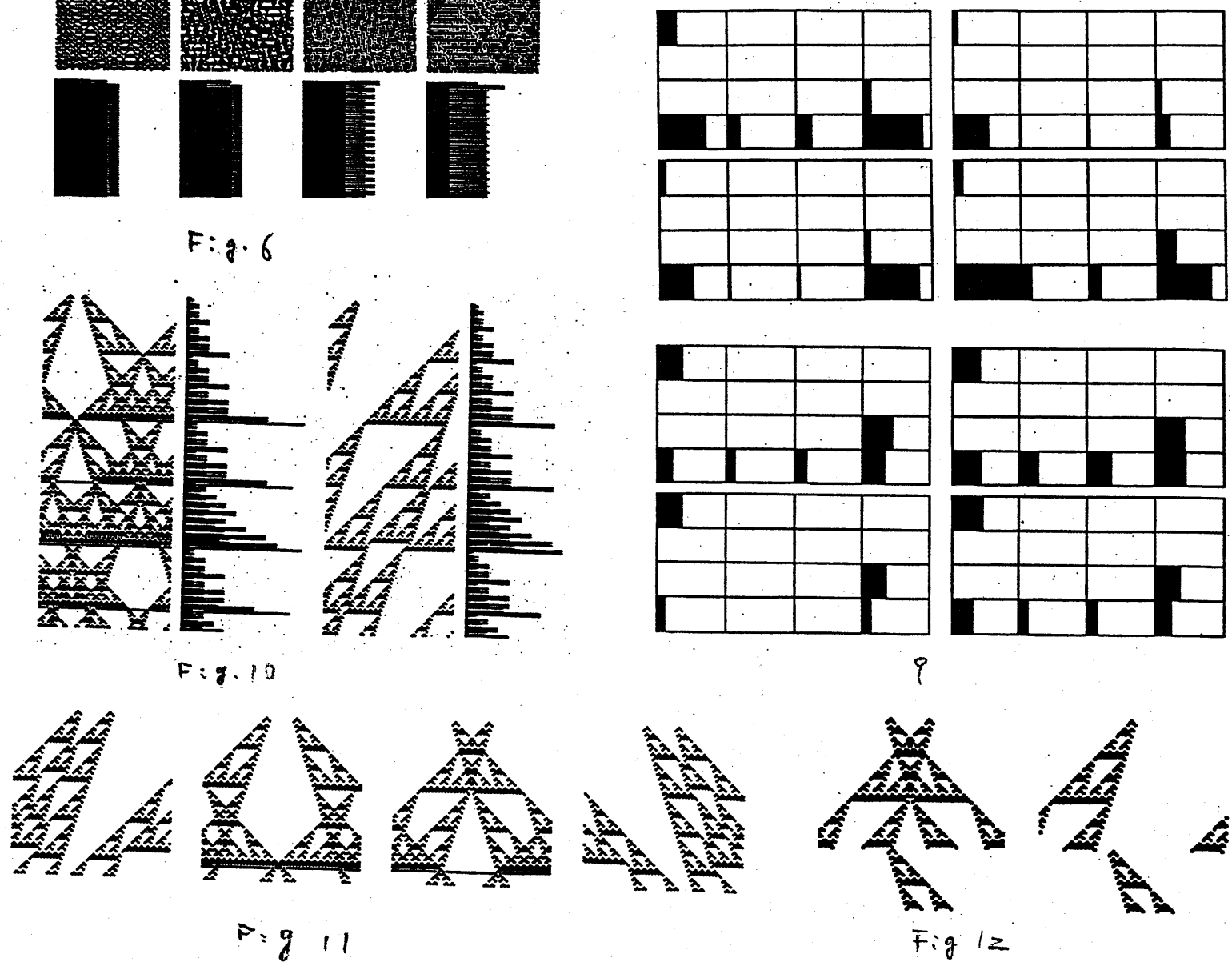

Fig 12 